\title{
On the Gibbs phase rule in the Pirogov-Sinai regime
}

\author{
A. Bovier, I. Merola, E. Presutti, and M. Zahradník
}

\begin{abstract}
We consider extended Pirogov-Sinai models including lattice and continuum particle systems with Kac potentials. Calling $\lambda$ an intensive variable conjugate to an extensive quantity $\alpha$ appearing in the Hamiltonian via the additive term $-\lambda \alpha$, we prove that if a Pirogov-Sinai phase transition with order parameter $\alpha$ occurs at $\lambda=0$, then this is the only point in an interval of values of $\lambda$ centered at 0 , where phase transitions occur.
\end{abstract}

\section{Introduction}

"In the abstract space of all potentials, phase transitions are an exception". This statement by Ruelle in his classical textbook, [10], suggests the validity of the Gibbs phase rule, but the notion must be accepted only very cautiously, as remarked by van Enter, [5], [14] and then by Sokal and Israel, [13], [7]; anyway a complete proof of the Gibbs rule would require to show that in the space of the few thermodynamically relevant parameters, phase transitions occur on regular manifolds of positive co-dimension. But, as stated again by Ruelle in a recent review on open problems in mathematical physics, [11], the proof of such a statement must be regarded as one of the main challenges in statistical mechanics.

In the Pirogov-Sinai regime where configurations can be described by contours which satisfy Peierls conditions, the situation is definitely better, as the theory provides tools for a very detailed knowledge on the structure of Gibbs measures in a region in the relevant parameters space; see for instance Sinai's book on phase transitions, [12]. The traditional Pirogov-Sinai theory is a low temperature expansion which enables to control the entropic fluctuations from the ground states, its natural setup being the lattice systems. But the theory is not limited to such cases and it has been applied to a great variety of situations,

Research partially supported by the DFG Research network 1033.

Research partially supported by MURST and NATO Grant PST.CLG.976552 and the DFG Research network 1033.

Research partially supported by NATO Grant PST.CLG.976552 and GAČR 201/03/0478 . 
covering various types of phase transitions. We just mention here the case of Kac potentials, which are seen as a perturbation of mean field, where the small parameter is the inverse interaction range of a Kac potential. According to van der Waals, the theory becomes then well suited for investigating the liquid-vapor branch of the phase diagram and, as shown in [9], its applications are not restricted to lattice models, [4], [2], but continuum particle systems can be treated as well.

All the above cases have a common structure. There is a term in the Hamiltonian of the form $-\lambda \alpha$, where $\alpha$ is an extensive quantity and $\lambda \in \mathbb{R}$ is its conjugate variable: in the case of spins $\lambda$ is an external magnetic field and $\alpha$ the spin magnetization; for particles, $\lambda$ is the chemical potential and $\alpha$ the particles number. Our main assumption is that at a value, say $\lambda=0$, of the intensive parameter there is phase coexistence with $\alpha$ an order parameter, and that defining contours in terms of the variable $\alpha$, the contours satisfy the Peierls bounds with suitable coefficients. Under this assumption (plus some technical conditions of superstability type if the variables are unbounded) we prove that there is a finite interval of values of $\lambda$, centered at $\lambda=0$, where coexistence occurs only at $\lambda=0$. The proof does not need to specify the precise structure of $\alpha$, nor the precise definition of contours and it covers in a unified way both perturbations of ground states and Kac potentials. The proof is truly simple, at least we hope, and it avoids the traditional requirements about the structure of the restricted ensembles free energies, which in the applications are not always easy to verify. A more detailed (and maybe more model dependent) analysis is needed if we want to investigate finer questions as differentiability or analytic continuation. As shown recently by Friedli and Pfister[6], also for such questions the analysis extends from the traditional setup of the Pirogov-Sinai regime to models with Kac potentials.

In Section 2 we specify the setup and state the main results. In Section 3 we recall the Peierls argument to prove the occurrence of a phase transition at $\lambda=0$. Section 4

is the most original part of the paper, we prove there that if $\lambda$ is positive or negative, then, correspondingly, the plus or the minus phases are stable, namely Peierls bounds hold true for the corresponding contours. In Section 5, we complete the analysis by showing uniqueness. In Section 6 we prove that the assumptions in Section 2 are verified in the LMP model for phase transitions in the continuum.

\section{Model and main results}

\section{State space}

After partitioning the physical space $\left(\mathbb{Z}^{d}\right.$ or $\left.\mathbb{R}^{d}, d \geq 2\right)$ into cells made of cubes of side $\ell$ ( $\ell$ a free parameter whose choice will be discussed later), the system becomes a lattice 
model with a general single site state space $Q$. In this way we will treat in a uniform way models on a lattice and in the continuum. The main examples we have in mind are finite spins and point particles:

$$
Q:=\left\{\begin{array}{l}
\{-k, \ldots, k\}^{\ell^{d}} \quad \text { with } k \text { and } \ell \text { positive integers } \\
\left(n ; r_{1}, \ldots, r_{n}\right) \quad n \in \mathbb{N}, r_{i} \in(-\ell / 2, \ell / 2]^{d}, \ell>0
\end{array}\right.
$$

In general, $Q$ is a polish space, whose generic element is $q$ while $\|q\|$ denotes its norm; a configuration is then $q:=\left\{q_{x}\right\}_{x \in \mathbb{Z}^{d}}$, which is an element of the phase space $Q^{\mathbb{Z}^{d}}, q_{x}$ being the configuration at $x \in \mathbb{Z}^{d}$. In the particles case, $q_{x}:=\left(n ; r_{1}, \ldots, r_{n}\right)$ is the collection of positions of the particles which are in the cell $C_{x}$. We equip the space $Q^{\mathbb{Z}^{d}}$ with the product topology corresponding tothe metric topology on $Q$. Of course this topology is metrizable with a norm given e.g. by $\|q\|=\sum_{x \in \mathbb{Z}^{d}} 2^{-|x|}\left\|q_{x}\right\|$, where $|x|=\sup _{i \in 1, \ldots, d}\left|x_{i}\right|$. In the sequel we will call two sites, $x$ and $y$, connected if $|x-y| \leq 1$, thus in this paper connected sets are the same as $*$ connected sets.

\section{Free measure}

We consider a fixed probability measure on $Q$ (the counting measure or the law of a Poisson process, in above examples). The free measure is then the product measure $\nu$ on $Q^{\mathbb{Z}^{d}} \cdot \nu_{\Lambda}(\cdot)$ denotes the marginal of $\nu(d q)$ on $Q^{\Lambda}$ for $\Lambda \subset \mathbb{Z}^{d}$.

The models we consider are defined by Hamiltonians obtained in the usual way from interaction potentials. We will always assume translational invariance, finite range and continuity of the potentials, which are then bounded when $Q$ is compact. In the non-compact case, we allow for unbounded potentials, supposing they give rise to Gibbs measures $\mu$ with the property that the interactions remain almost surely bounded.

\section{Unperturbed Hamiltonian and contours}

We fix a reference Hamiltonian $H_{0}$ which incorporates the inverse temperature $\beta$ as a factor, so that $H_{0}$ is dimensionless. We define $H_{0}$ by giving the family of its potentials $\left\{U_{\Delta}\right\}$, recalling that the energy of a configuration $q_{\Lambda}$ in the finite region $\Lambda$ in interaction with the configuration $p$ in the complement of $\Lambda$ is given by the formula

$$
H_{0}\left(q_{\Lambda} \mid p_{\Lambda^{c}}\right) \equiv \sum_{\Delta \sqcap \Lambda \neq \emptyset} U_{\Delta}\left(q_{\Lambda}, p_{\Lambda^{c}}\right)
$$

where $\left(q_{\Lambda}, p_{\Lambda^{c}}\right)$ is the configuration which agrees with $q_{\Lambda}$ and $p_{\Lambda^{c}}$ respectively on $\Lambda$ and $\Lambda^{c}$. We choose the cell length $\ell$ in such a way that $U_{\Delta} \equiv 0$ unless $\Delta$ is a translate of the cube $|x| \leq 1$. Abusing notation we then write $U_{x}$ for the potential relative to the cube $\Delta$ 
with center at $x$. We then have

$$
H_{0}\left(q_{\Lambda} \mid p_{\Lambda^{c}}\right)=\sum_{x: \operatorname{dist}(x, \Lambda) \leq 1} U_{x}\left(\left(q_{\Lambda}, p_{\Lambda^{c}}\right)\right)
$$

We suppose that $U_{x}(q)$ is a translation invariant function, i.e.

$$
U_{x}(q)=U_{0}\left(\tau_{x} q\right), \quad\left(\tau_{x} q\right)_{y}=q_{x+y}
$$

with $U_{0}(q)$ depending only on $\left\{q_{y},|y| \leq 1\right\}$. The other assumptions on $H_{0}$ will be stated later.

We are going to describe the configurations in terms of contours, and, as we will see later, the validity of a Peierls condition for $H_{0}$ will constitute the main assumption on the Hamiltonian. By adjusting the choice of $\ell$, contours are simply described in the following way. There is a single spin function $\theta\left(q_{x}\right)$ with values in $\{0, \pm 1\}$, so that the phase variable

$$
\Theta(q ; x)=\prod_{y:|y-x| \leq 1} \mathbf{1}_{\theta\left(q_{y}\right)=1}-\prod_{y:|y-x| \leq 1} \mathbf{1}_{\theta\left(q_{y}\right)=-1}
$$

indicates the spatial distribution of phases: namely the regions $\{\Theta(q ; x)= \pm 1\}$ are respectively called the \pm equilibrium regions, while the maximal connected components of $\{\Theta(q ; x)=0\}$, denoted by $\operatorname{sp}\left(\Gamma_{i}\right)$, are the spatial supports of the contours. A contour $\Gamma$ is then the pair $\Gamma=\left(\operatorname{sp}(\Gamma), \theta_{\Gamma}\right)$, where $\theta_{\Gamma}$ is the restriction of $\theta$ to $\operatorname{sp}(\Gamma)$.

In the sequel all configurations $q$ that appear as boundary conditions will be assumed to be such that the set of points $x \in \mathbb{Z}^{d}$ where $\theta\left(q_{x}\right) \neq 0$ is compact.

\section{The full Hamiltonian}

The full Hamiltonian that we consider has the expression:

$$
H_{\lambda}(q)=H_{0}(q)+\lambda H_{I}(q)
$$

where $\lambda H_{I}$ is the perturbation and $\lambda$ the coupling strength. The main examples we have in mind are when $\lambda$ is a magnetic field and $-H_{I}(q)$ the magnetization density, and when $\lambda$ is the chemical potential while $-H_{I}(q)$ is the particles density. More generally and analogously to (2.1) we set

$$
H_{I}\left(q_{\Lambda} \mid p_{\Lambda^{c}}\right)=-\left(\beta \ell^{d}\right) \sum_{x: \operatorname{dist}(x, \Lambda) \leq 1} \alpha_{x}\left(\left(q_{\Lambda}, p_{\Lambda^{c}}\right)\right)
$$

with $\alpha_{x}(q)=\alpha_{0}\left(\tau_{x} q\right)$ dependent only on $q_{y},|y-x| \leq 1$ (after a proper choice of the cell length $\ell$ ). Thus, in the above examples, $\alpha_{x}(q)$ is respectively the magnetization and the particles densities in the cell indexed by $x$.

The coefficient $\beta \ell^{d}$ could be incorporated in $\lambda$, but we prefer to have the two quantities separated, to keep a connection with the original model, before its representation in cells. 
We will suppose that $\alpha$ is an order parameter for $H_{0}$, namely that there are $\alpha_{+}>\alpha_{-}$ so that

$$
\Theta(q ; x)= \pm 1 \Longrightarrow\left|\alpha_{x}(q)-\alpha_{ \pm}\right| \leq \zeta, \quad \zeta=\frac{\alpha_{+}-\alpha_{-}}{10}
$$

(any other choice with $\zeta<\left(\alpha_{+}-\alpha_{-}\right)$would work as well).

\section{Gibbs and DLR measures}

The Gibbs measure $\mu_{\lambda, \Lambda}^{(p)}(d q)$ on the finite region $\Lambda \subset \mathbb{Z}^{d}$ with b.c. $p$ is given by

$$
\mu_{\lambda, \Lambda}^{(p)}(d q):=\frac{e^{-H_{\lambda}\left(q_{\Lambda} \mid p_{\Lambda^{c}}\right)}}{Z_{\lambda, \Lambda}^{(p)}} \nu_{\Lambda}\left(d q_{\Lambda}\right) \delta_{p_{\Lambda^{c}}}\left(d q_{\Lambda^{c}}\right)
$$

The denominator $Z_{\lambda, \Lambda}^{(p)}$ is the partition function.

$$
Z_{\lambda, \Lambda}^{(p)}:=\int \nu_{\Lambda}\left(d q_{\Lambda}\right) \delta_{p_{\Lambda^{c}}}\left(d q_{\Lambda^{c}}\right) e^{-H_{\lambda}\left(q_{\Lambda} \mid p_{\Lambda^{c}}\right)}
$$

and

$$
H_{\lambda}\left(q_{\Lambda} \mid p_{\Lambda^{c}}\right)=\sum_{x: \operatorname{dist}(x, \Lambda) \leq 1}\left(U_{x}\left(\left(q_{\Lambda}, p_{\Lambda^{c}}\right)\right)-\lambda\left(\beta \ell^{d}\right) \alpha_{x}\left(\left(q_{\Lambda}, p_{\Lambda^{c}}\right)\right)\right)
$$

A DLR measure $\mu(d q)$ is then defined by the local specifications $\mu_{\lambda, \Lambda}^{(p)}(d q)$, by requiring that the conditional probability of $\mu(d q)$ on the $\sigma$-algebra $\mathcal{F}_{\Lambda^{c}}$ generated by $\left\{q_{x}, x \in \Lambda^{c}\right\}$, is $\mu$ a.s. equal to $\mu_{\lambda, \Lambda}^{(p)}(d q)$.

The \pm Gibbs measures.

The configuration $p$ is a \pm boundary condition w.r.t. the finite region $\Lambda \sqsubset \mathbb{Z}^{d}$, if $\theta\left(p_{x}\right) \equiv \pm 1$ for all $x \in \Lambda^{c}$ such that $\operatorname{dist}(x, \Lambda) \in\{1,2\}$. Then the \pm finite volume, Gibbs measures $\mu_{\lambda, \Lambda}^{( \pm, p)}$ are

$$
\mu_{\lambda, \Lambda}^{( \pm, p)}(d q):=\frac{e^{-H_{\lambda}\left(q_{\Lambda} \mid p_{\Lambda^{c}}\right)}}{Z_{\lambda, \Lambda}^{( \pm, p)}} \mathbf{1}_{\{\Theta(q, x)= \pm 1, \operatorname{dist}(x, \Lambda)=1\}} \nu_{\Lambda}\left(d q_{\Lambda}\right) \delta_{p_{\Lambda^{c}}}\left(d q_{\Lambda^{c}}\right)
$$

where the partition function $Z_{\lambda, \Lambda}^{( \pm, p)}$ is defined as the normalization factor for $\mu_{\lambda, \Lambda}^{( \pm, p)}(d q)$ to be a probability.

\section{The Peierls condition}

We will say that the Peierls condition holds with constant $C_{P}>0, P$ standing for Peierls, if for any finite region $\Lambda$, any \pm boundary condition $p$ and any sequence $\Gamma_{1}, \ldots, \Gamma_{m}$ of contours with $\operatorname{sp}\left(\Gamma_{i}\right) \sqsubset \Lambda$,

$$
\mu_{0, \Lambda}^{( \pm, p)}\left(q: \Gamma_{1}, \ldots, \Gamma_{m} \text { are contours of } q\right) \leq e^{-C_{P} \sum_{i}\left|\Gamma_{i}\right|}
$$


where we have denoted by $|\Gamma|:=|\operatorname{sp}(\Gamma)|$.

Notice that the condition refers only to $\lambda=0$. It will be used under the assumption that $C_{P}$ is large: that is, $C_{P}$ larger than some universal, dimension dependent constant. Under such an assumption, (2.9) implies the occurrence of a phase transition, as stated in Theorem 2.1.

The condition $C_{P}$ large is in general model dependent, and our point here is that once and for whatever reason, the condition is checked to hold, then all the properties we will state below are valid and can be applied.

In traditional Pirogov-Sinai models the condition that $C_{P}$ is large is verified when $\beta$ is large, while in the case of Kac potentials it follows from assuming the scaling parameter $\gamma$ small. In both cases $\left(\beta \ell^{d}\right)$ large is the condition responsible for the largeness of $C_{P}$. While $C_{P}$ diverges with $\left(\beta \ell^{d}\right)$, in general the ratio $C_{P} /\left(\beta \ell^{d}\right)$ is infinitesimal and the range of values of the coupling constant $\lambda$ will be determined by the such a ratio

$$
\delta:=\frac{C_{P}}{\beta \ell^{d}}
$$

\section{Temperedness conditions}

We suppose that there are constants $c_{n}, c_{n}^{\prime}, n \in \mathbb{N}$, so that

$$
\begin{gathered}
\mu_{\lambda, \Lambda}^{\left(\left\{\epsilon_{i}\right\}, p\right)}\left(\left|\alpha_{x}(q)\right|^{n}\right) \leq c_{n}, \quad \text { for any } x \in \Lambda \\
\mu_{\lambda, \Lambda}^{\left(\left\{\epsilon_{i}\right\}, p\right)}\left(q:\left|q_{x}\right| \geq t\right) \leq c_{n}^{\prime} t^{-n}, \quad \text { for any } x \in \Lambda \text { and } t>1
\end{gathered}
$$

and call "tempered" a probability on $Q^{\mathbb{Z}^{d}}$ such that, for some coefficient $c_{n}^{\prime}$,

$$
\mu\left(q:\left|q_{x}\right| \geq t\right) \leq c_{n}^{\prime} t^{-n}, \quad \text { for any } x \in \mathbb{Z}^{d} \text { and } t>1
$$

\section{Large deviations, Cut and paste bounds}

There is a positive function $C^{*}(a), a>0$, with the following property. Let $\Lambda$ be any finite region, $\left\{T_{i}\right\}$ the maximal, connected components of the complement of $\Lambda,\left\{\epsilon_{i}\right\}$ parameters with values in $\pm 1, \Delta$ any subset of $\Lambda$. Then, calling $\mu_{\lambda, \Lambda}^{\left(\left\{\epsilon_{i}\right\}, p\right)}$ the measure $\mu_{\lambda, \Lambda}^{p}$ conditioned on $\left\{\Theta(\cdot ; x)=\epsilon_{i}\right.$ on $\left.T_{i} \sqcap \operatorname{dist}(x, \Lambda)=1\right\}$,

$$
\mu_{\lambda, \Lambda}^{\left(\left\{\epsilon_{i}\right\}, p\right)}\left(\left|\alpha_{\Delta}(q)\right| \geq a|\Delta|\right) \leq e^{-\left(\beta \ell^{d}\right) C^{*}(a)|\Delta|}, \quad \alpha_{\Delta}=\sum_{x \in \Delta} \alpha_{x}
$$

Notice that the above condition is automatically satisfied when $Q$ is compact.

In Section 5 (when proving uniqueness), we will need the following cut and paste bound, namely that there are a constant $c>0$ and $n>0$ so that the following holds. Let $\Delta$ be 
a bounded region, $t>1, \bar{q}$ and $p$ two configurations such that, for any $x,\left|\bar{q}_{x}\right| \leq t$ and $\left|p_{x}\right| \leq t$. Then, for $\epsilon_{0}= \pm 1$,

$$
\frac{Z_{\lambda, \Delta}^{(\bar{q})}\left(\left|q_{x}\right| \leq t, x \in \Delta\right)}{Z_{\lambda, \Delta}^{(p)}\left(\theta\left(q_{x}\right)=\epsilon_{0}, x \in \Delta\right)} \leq e^{c\left(\beta \ell^{d}\right) t^{n}|\Delta|}
$$

where the argument of the partition function means that the partition function is defined with the constraint indicated by the argument.

The bound (2.15) could be proved as a consequence of assumptions on the interaction like

$$
\left|H_{\lambda, \Delta}\left(q_{\Delta} \mid \bar{q}_{\Delta^{c}}\right)\right|,\left|H_{\lambda, \Delta}\left(q_{\Delta} \mid p_{\Delta^{c}}\right)\right| \leq C_{I} t^{n}|\Delta|
$$

$\left(C_{I}\right.$ a suitable constant, proportional to $\beta$, while $n$ is determined by the form of the interaction: e.g. $n=2$ for pair interactions, $n=4$ in the LMP model) and on assumptions on the free measure of the sets $\theta= \pm 1$ :

$$
\int_{\theta\left(q_{x}\right)=\epsilon_{0}} \nu_{x}\left(d q_{x}\right) \geq e^{-c \ell^{d}}
$$

The sets $\mathcal{G}_{\lambda}^{ \pm}$

For any bounded set $\Lambda$ in $\mathbb{Z}^{d}$, we denote by $\mathcal{G}_{\lambda, \Lambda}^{+}$the weak closure of the family of measures which are convex combinations of the family $\left\{\mu_{\lambda, \Lambda_{i}}^{\left(+, p_{i}\right)}, \Lambda_{i} \sqsupseteq \Lambda\right\}$. $\mathcal{G}_{\lambda, \Lambda}^{-}$is defined analogously. Then, for any $\mu \in \mathcal{G}_{\lambda, \Lambda}^{ \pm}$,

$$
\mu\left(q:\left|q_{x}\right| \geq t\right) \leq c_{n}^{\prime} t^{-n}, \quad \text { for any } x \in \Lambda \text { and } t>1
$$

$\left(c_{n}^{\prime}\right.$ as in $\left.(2.12)\right)$. As a consequence, $\mathcal{G}_{\lambda}^{ \pm}$is weakly compact and since

$$
\mathcal{G}_{\lambda, \Lambda}^{ \pm} \sqsupseteq \mathcal{G}_{\lambda, \Delta}^{ \pm}, \quad \text { when } \Delta \sqsupseteq \Lambda
$$

we conclude that

$$
\mathcal{G}_{\lambda}^{+}=\sqcap \mathcal{G}_{\lambda, \Lambda}^{+}, \quad \mathcal{G}_{\lambda}^{-}=\sqcap \mathcal{G}_{\lambda, \Lambda}^{-}
$$

are both non empty, weakly compact, convex sets of tempered DLR measures. Notice that $\mathcal{G}_{\lambda}^{ \pm}$, which are both non empty, are however not necessarily distinct.

We can now state our main theorem.

Theorem 2.1. There are dimensions dependent constants $C_{P}(d), d \geq 2$, so that if $C_{P} \geq C_{P}(d)$, then:

- At $\lambda=0, \mathcal{G}_{0}^{+} \sqcap \mathcal{G}_{0}^{-}=\emptyset$ and if $\mu \in \mathcal{G}_{0}^{ \pm}$then, for all $x, \mu(\Theta(\cdot ; x)= \pm 1)>1 / 2$.

If, moreover, there is $a^{*}>0$ so that $\left(\beta \ell^{d}\right) C^{*}\left(a^{*}\right)>C_{P}$ (in the sequel we shorthand $C^{*}=C^{*}\left(a^{*}\right)$, the latter defined in (2.14)), then for suitable constants $C(d)>0$ : 
- If $0<\lambda \leq \lambda^{*}=C(d) \delta^{2}$, (resp. $0>\lambda \geq-\lambda^{*}$ ), any tempered DLR measure $\mu$ (see (2.13)) is in $\mathcal{G}_{\lambda}^{+}$(resp. $\left.\mathcal{G}_{\lambda}^{-}\right)$and, for all $x, \mu(\Theta(\cdot ; x)=1)>1 / 2(\operatorname{resp} . \mu(\Theta(\cdot ; x)=-1)>$ $1 / 2)$.

Theorem 2.1 will be proved in the remaining sections, in particular the statement at $\lambda=0$ is proved in Section 3, while the statements at $\lambda \neq 0$ are proved in Sections 4 and 5 .

Theorem 2.1 proves uniqueness in a "small" interval of values of $\lambda$, in particular in the case of Kac potentials the interval becomes infinitesimal as $\gamma \rightarrow 0, \gamma$ the Kac scaling parameter, see Section 6 for the LMP model. A different argument must be used for the larger $\lambda$, which in many applications (including Kac potentials and traditional PirogovSinai models) is based on the fact that the double well structure (of the energy or of the mean field free energy, for Kac potentials) at $\lambda=0$ is unbalanced when $\lambda \neq 0$. The one well case is in general "easy to treat" at least if the unbalance between wells is not too small, i.e. if $\lambda$ is large enough. Thus our theorem covers the dangerous case of the small perturbations. The extension to larger $\lambda$ involves new assumptions on the structure of the Hamiltonian and seems more model dependent. In separate papers some specific cases will be treated.

\section{Phase transitions and the Peierls argument}

In this section we will prove the statement of Theorem 2.1 about the occurrence of a phase transition at $\lambda=0$. The proof is nothing but the classical Peierls argument and we report it mainly because it introduces notions that will be used in the sequel.

\section{Geometry of contours.}

Given a contour $\Gamma$, we denote by $\operatorname{ext}(\Gamma)$ the unbounded maximal connected component of $\operatorname{sp}(\Gamma)^{c}$. The contour is called a $+[-]$ contour if $\theta_{\Gamma}(x)=1$ [resp. $\left.=-1\right]$ for all $x$ such that $\operatorname{dist}(x, \operatorname{ext}(\Gamma))=1$. By definition of contours $\theta_{\Gamma}$ is constant on $\{x: \operatorname{dist}(x, \operatorname{ext}(\Gamma))=1\}$ and different from 0 . We call $\operatorname{int}(\Gamma)=\operatorname{sp}(\Gamma)^{c} \backslash \operatorname{ext}(\Gamma)$, and distinguish $\operatorname{int}^{ \pm}(\Gamma)$ as the union of 
all the maximal connected components of int $(\Gamma)$ which are connected to regions where $\theta_{\Gamma}$ is respectively \pm 1 . Finally we call

$$
V(\Gamma)=\operatorname{sp}(\Gamma) \sqcup \operatorname{int}(\Gamma)
$$

\section{Mass of contours.}

The mass of a \pm contour $\Gamma$ relative to a probability $\mu$ on $Q^{\mathbb{Z}^{d}}$, is defined for all configurations $p$ which are respectively \pm b.c. relative to the region $V(\Gamma)$, as

$$
\mu(\Gamma ; p):=\mu\left(q: \Gamma \text { is a } \pm \text { contour for } q \mid \mathcal{F}_{V(\Gamma)^{c}} ;\{\Theta(q ; x)= \pm 1, \operatorname{dist}(x, V(\Gamma))=1\}\right)(p)
$$

namely $\mu(\Gamma ; p)$ is the conditional probability computed at $p$ of having the contour $\Gamma$, given the $\sigma$-algebra generated by the variables $q_{x}, x \in V(\Gamma)^{c}$ and given that $\{\Theta(q ; x)=$ $\pm 1, \operatorname{dist}(x, V(\Gamma))=1\}$.

If $\Gamma$ is a \pm contour and $\mu=\mu_{\lambda, V(\Gamma)}^{( \pm, p)}$, we write its mass as $m_{\lambda}(\Gamma ; p)$, namely

$$
m_{\lambda}(\Gamma ; p):=\mu_{\lambda, V(\Gamma)}^{( \pm, p)}(q: \Gamma \text { is a contour for } q)
$$

which, sometimes, will be simply called "the mass of the contour $\Gamma$ ".

Notice that a different notion is often used in the literature, namely that of "the weight of a contour", which is defined in terms of a ratio of two partition functions, one with the constraint of having a contour $\Gamma$ and the other one with the constraint that the contour $\Gamma$ is absent.

Finally, $\mu$ satisfies the Peierls condition relative to a class $\{\Gamma\}$ of contours and with constant $C$, if

$$
\mu(\Gamma ; p) \leq e^{-C|\Gamma|} \text { for all contours } \Gamma \in\{\Gamma\} \text { and all } p
$$

Lemma 3.1. If $C$ is large enough, then

$$
\sum_{\Gamma: 0 \in V(\Gamma)} e^{-C|\Gamma|} \leq e^{-C}
$$

The proof starts from the observation that $|\Gamma| \geq 7$ (in $d=2$ the minimal contour consists of a site and those connected to it). Thus the sum in (3.5) starts from sets with at least 7 sites. We can then factorize a term $e^{-C}$ and need to prove that the remaining sum is bounded by 1, if $C$ is large enough. This involves a counting argument which, being by now classical, is omitted. 
Proof of the statement in Theorem 2.1 at $\lambda=0$.

Suppose the measures $\mu_{0, \Lambda}^{( \pm, p)}$ satisfy (3.4) with $C>1$ and as large as required by Lemma 3.1. Then, for any $x \in \Lambda$ :

$$
\mu_{0, \Lambda}^{( \pm, p)}\left(\left|\alpha_{x}-\alpha_{ \pm}\right|>\zeta\right)=\mu_{0, \Lambda}^{( \pm, p)}(x \in V(\Gamma), \text { for some } \Gamma) \leq 1 / e
$$

The inequality remains valid after convex combinations and weak limits, proving that $\mathcal{G}_{0}^{ \pm}$ are distinct.

\section{Some generalizations.}

In the next section we will use variants of the above argument which are stated below. We will denote by $\mu(f)$ the expectation of a function $f$, relative to a measure $\mu$.

Lemma 3.2. Let $\mu$ be supported by configurations $q$ having $\Theta(q ; x)=\epsilon_{0}, \epsilon_{0} \in\{ \pm 1\}$, for all $x \in \Lambda^{c}, \Lambda$ a bounded region. Then, if $\mu$ satisfies the Peierls bounds for all $\epsilon_{0}$-contours with a constant $C$ as large as needed in Lemma 3.1, for any $x \in \Lambda$

$$
\begin{gathered}
\mu\left(\left|\alpha_{x}-\alpha_{\epsilon_{0}}\right|\right) \leq \zeta+e^{-C / 2} \mu\left(\left|\alpha_{x}-\alpha_{\epsilon_{0}}\right|^{2}\right)^{1 / 2} \\
\mu\left(\left|\alpha_{x}-\alpha_{\epsilon_{0}}\right| \mathbf{1}_{\left|\alpha_{x}-\alpha_{\epsilon_{0}}\right|>\zeta}\right) \leq e^{-C / 2} \mu\left(\left|\alpha_{x}-\alpha_{\epsilon_{0}}\right|^{2}\right)^{1 / 2}
\end{gathered}
$$

Proof. We partition the space into the sets $\left|\alpha_{x}-\alpha_{\epsilon_{0}}\right| \leq \zeta$ and $\left|\alpha_{x}-\alpha_{\epsilon_{0}}\right|>\zeta$. The contribution to the expectation (3.7) of the first set is bounded by $\zeta$. All configurations in the support of $\mu$ which belong to the second set, have a $\epsilon_{0}$-contour $\Gamma$ with $x \in V(\Gamma)$. We then use Cauchy-Schwartz and (3.5) to prove (3.7) and (3.8).

\section{Stability of phases}

In this section we will prove that for $0<\lambda \leq \lambda^{*}$,

$$
m_{\lambda}(\Gamma ; q) \leq e^{-\left(C_{P} / 8\right) N_{\Gamma}}, \quad \text { for any }+ \text { contour } \Gamma
$$

and that the analogous property holds for negative $\lambda$ and - contours. 
By standard arguments and using the analysis of Section 3 (details are omitted), it follows from (4.1) that for $C_{P}$ large enough, if $\lambda>0[\lambda<0]$, then any measure in $\mathcal{G}^{+}$[in $\left.\mathcal{G}^{-}\right]$is supported by configurations $q$ such that the set $\{x: \Theta(q, x)=1\}[\{x: \Theta(q, x)=-1\}]$ occupies most of the space. The persistence of this property past $\lambda=0$ is usually referred to as "a stability of the $+($ or - ) phase" under the perturbation of strength $\lambda$.

To have lighter notation we restrict in the sequel to positive $\lambda$, the proofs for negative $\lambda$ being completely similar are omitted. (4.1) will follow from showing that the typical configurations of $\mu_{\lambda, \Lambda}^{(-, p)}$ have a layer close to the boundary of $\Lambda$, where mostly $\Theta=-1$, but after the layer typically $\Theta=1$. An improved control on the thickness of the layer and on the absence of finger-like protuberances will prove in Section 5 that any tempered DLR measure is in $\mathcal{G}^{+}$.

The key point is to show that $\Theta=-1$ has a cost and that it is therefore convenient to have a transition to $\Theta=1$. The cost comes from the energy $-\lambda \alpha(q)$ which would decrease from erasing the minus layer and thus changing $\Theta=-1$ into $\Theta=1$. The problem comes from the entropic factor due to the number of ways to realize the minus layer attached to the boundary of $\Lambda$. If $\lambda$ is very small, such an entropic factor becomes too large and the layer impossible to erase. We will fix the problem by not regarding as being in the plus phase any region with $\Theta=1$ whose volume is "too small": the entropy is then drastically reduced and under control. With this in mind we introduce the notion of

$$
\text { "Slim" and "Fat" contours }
$$

Slim and fat contours are distinguished using the length scale

$$
\xi_{-}(\lambda):=\lambda^{-1 / 2}
$$

recall that we are supposing $\lambda>0$ small and therefore $\xi_{-}(\lambda)$ is a large quantity. The choice of the power $1 / 2$ in the above definition is to a large extent arbitrary.

Definition 4.1. A contour $\Gamma$ is called " $\lambda$-slim" if

$$
|V(\Gamma)| \leq \xi_{-}(\lambda)^{d}
$$

where $V(\Gamma)$ is defined in (3.1). A contour that is not $\lambda$-slim is called " $\lambda$-fat". Similarly, a region $\Lambda$ is called " $\lambda$-fat" if it is a bounded region, such that all the maximal connected components of $\Lambda^{c}$ have volume larger than $\xi_{-}(\lambda)^{d}$.

Remark: When there is no risk of confusion, we will simply say "slim" and "fat" contours.

Proposition 4.2. For $0<\lambda \leq \lambda^{*}$ the mass of any slim contour $\Gamma$ is bounded by

$$
m_{\lambda}(\Gamma ; q) \leq e^{-\left(C_{P} / 2\right)|\Gamma|} \leq e^{-\left(C_{P} / 4\right)|\Gamma|-\lambda\left(\beta \ell^{d}\right)\left(\alpha_{+}-\alpha_{-}\right)|V(\Gamma)|}
$$


(the latter inequality will be used at the end of Section 5). The analogous statement holds for $-\lambda^{*} \leq \lambda<0$.

Proof. For notational simplicity we will refer to $\lambda$ positive and + contours. We will denote by $Z(A)$ a partition function with the constraint that the configurations should belong to the set $A$. We then have for a $+\operatorname{contour~} \Gamma$,

$$
m_{\lambda}(\Gamma ; p)=\frac{Z_{\lambda, V(\Gamma)}^{(+, p)}(\Gamma \text { is a contour })}{Z_{\lambda, V(\Gamma)}^{(+, p)}}
$$

Then, by (2.14) and recalling the definition of $a^{*}$ in Theorem 2.1,

$$
\begin{aligned}
Z_{\lambda, V(\Gamma)}^{(+, p)}(\Gamma \text { is a contour }) \leq & Z_{\lambda, V(\Gamma)}^{(+, p)}\left(\Gamma \text { is a contour; }\left|\alpha_{V(\Gamma)}\right| \leq a^{*}|V(\Gamma)|\right) \\
& +e^{-C_{P}|\Gamma|} Z_{\lambda, V(\Gamma)}^{(+, p)}
\end{aligned}
$$

In the first partition function on the r.h.s. we want to replace $\lambda$ by 0 , in order to use the Peierls bounds valid by assumption at $\lambda=0$. We have

$$
H_{\lambda}\left(q_{V(\Gamma)} \mid q_{V(\Gamma)^{c}}\right)-H_{0}\left(q_{V(\Gamma)} \mid q_{V(\Gamma)^{c}}\right)=-\lambda\left(\beta \ell^{d}\right) \sum_{\operatorname{dist}(x, V(\Gamma)) \leq 1} \alpha_{x}(q)
$$

On the other hand, due to the constraints in the partition function, we can restrict to configurations $q$ which agree with $p$ on $\Lambda^{c}$, and such that both $\left|\alpha_{V(\Gamma)}\right| \leq a^{*}|V(\Gamma)|$ and $\Theta(q ; x)=1$ when $\operatorname{dist}(x, V(\Gamma))=1$. Calling $\left|\alpha_{ \pm}\right|=\max \left\{\left|\alpha_{+}\right|,\left|\alpha_{-}\right|\right\}$, we have

$$
\begin{aligned}
& \left|H_{\lambda}\left(q_{V(\Gamma)} \mid q_{V(\Gamma)^{c}}\right)-H_{0}\left(q_{V(\Gamma)} \mid q_{V(\Gamma)^{c}}\right)\right| \leq X \\
& X:=|\lambda|\left\{\left(\beta \ell^{d}\right) a^{*}|V(\Gamma)|+\left(2\left|\alpha_{ \pm}\right|\right)(2 d)|\Gamma|\right\}
\end{aligned}
$$

because the cardinality of $\{x: \operatorname{dist}(x, V(\Gamma))=1\}$ is bounded by $2 d|\Gamma|$ and $\left|\alpha_{+} \pm \zeta\right| \leq 2\left|\alpha_{ \pm}\right|$. Then

$$
\begin{gathered}
Z_{\lambda, V(\Gamma)}^{(+, p)}(\Gamma \text { is a contour }) \leq e^{X} Z_{0, V(\Gamma)}^{(+, p)}(\Gamma \text { is a contour })+e^{-C_{P}|\Gamma|} Z_{\lambda, V(\Gamma)}^{(+, p)} \\
\leq e^{X-C_{P}|\Gamma|} Z_{0, V(\Gamma)}^{(+, p)}+e^{-C_{P}|\Gamma|} Z_{\lambda, V(\Gamma)}^{(+, p)}
\end{gathered}
$$

and, writing $Z_{0, V(\Gamma)}^{(+, p)} \leq Z_{0, V(\Gamma)}^{(+, p)}\left(\left|\alpha_{V(\Gamma)}\right| \leq a^{*}|V(\Gamma)|\right)+e^{-C_{P}|\Gamma|} Z_{0, V(\Gamma)}^{(+, p)}$, we get

$$
\begin{aligned}
& \leq \frac{e^{X-C_{P}|\Gamma|}}{1-e^{-C_{P}|\Gamma|}} Z_{0, V(\Gamma)}^{(+, p)}\left(\left|\alpha_{V(\Gamma)}\right| \leq a^{*}|V(\Gamma)|\right)+e^{-C_{P}|\Gamma|} Z_{\lambda, V(\Gamma)}^{(+, p)} \\
& \leq\left(\frac{e^{2 X-C_{P}|\Gamma|}}{1-e^{-C_{P}|\Gamma|}}+e^{-C_{P}|\Gamma|}\right) Z_{\lambda, V(\Gamma)}^{(+, p)} \\
& \leq\left(\frac{e^{2 X-C_{P}|\Gamma| / 2}}{1-e^{-C_{P}|\Gamma|}}+e^{-C_{P}|\Gamma| / 2}\right) e^{-C_{P}|\Gamma| / 2} Z_{\lambda, V(\Gamma)}^{(+, p)}
\end{aligned}
$$


To bound the parameter $X$ introduced in (4.6), we use an isoperimetric inequality: there is $c_{d}>0$ such that

$$
|V(\Gamma)|^{(d-1) / d} \leq c_{d}|\Gamma|, \quad|V(\Gamma)| \leq c_{d}|\Gamma| \xi_{-}(\lambda)
$$

Then, recalling (2.10),

$$
X \leq\left(\left(C_{P} \delta^{-1} a^{*} c_{d}\right)\left|\lambda \xi_{-}(\lambda)\right|+\left(2\left|\alpha_{ \pm}\right|\right)(2 d)|\lambda|\right)|\Gamma|
$$

By the choice of $\lambda^{*}$ in Theorem 2.1, $\lambda \xi_{-}(\lambda) \leq \sqrt{\lambda^{*}} \leq \delta \sqrt{C(d)}$, so that the first inequality in (4.4) follows for $C(d)$ small enough and $C_{P}$ large enough.

To complete the proof of (4.4) we need to show that

$$
\lambda\left(\beta \ell^{d}\right)\left(\alpha_{+}-\alpha_{-}\right)|V(\Gamma)| \leq\left(C_{P} / 4\right)|\Gamma|
$$

By (4.7),

$$
\lambda\left(\beta \ell^{d}\right)\left(\alpha_{+}-\alpha_{-}\right)|V(\Gamma)| \leq \lambda\left(\beta \ell^{d}\right)\left(\alpha_{+}-\alpha_{-}\right) c_{d}|\Gamma| \xi_{-}(\lambda)
$$

hence (4.8), for $C(d)$ small enough.

For later reference we state a property analogous to one established in the proof of Proposition 4.2 and which follows directly from (2.14). Let $\Gamma$ be a contour and $p$ a configuration which has $\Gamma$ as a contour. Call $D=\left\{x: \operatorname{dist}(x, \operatorname{sp}(\Gamma)=1\}\right.$, then $\theta\left(p_{x}\right)$ is constant on each one of the connected components of $D$, and we denote by $\epsilon_{i}= \pm 1$ such values. Then, supposing $C_{P}$ so large that

$$
\begin{gathered}
1-e^{-C_{P}} \geq e^{-1}, \quad 1+c e^{-C_{P} / 4} \leq e \\
Z_{\lambda, \mathrm{sp}(\Gamma)}^{\left(\left\{\epsilon_{i}\right\}, p\right)}\left(\left|\alpha_{\mathrm{sp}(\Gamma)}\right|>a^{*}|\Gamma|\right) \leq e^{-C_{P}|\Gamma|+1} Z_{\lambda, \mathrm{sp}(\Gamma)}^{\left(\left\{\epsilon_{i}\right\}, p\right)}\left(\left|\alpha_{\mathrm{sp}(\Gamma)}\right| \leq a^{*}|\Gamma|\right)
\end{gathered}
$$

In fact, by (2.14),

$$
Z_{\lambda, \mathrm{sp}(\Gamma)}^{\left(\left\{\epsilon_{i}\right\}, p\right)}\left(\left|\alpha_{\mathrm{sp}(\Gamma)}\right|>a^{*}|\Gamma|\right) \leq e^{-C_{P}|\Gamma|} Z_{\lambda, \mathrm{sp}(\Gamma)}^{\left(\left\{\epsilon_{i}\right\}, p\right)}
$$

We then write

$$
Z_{\lambda, \mathrm{sp}(\Gamma)}^{\left(\left\{\epsilon_{i}\right\}, p\right)}=Z_{\lambda, \mathrm{sp}(\Gamma)}^{\left(\left\{\epsilon_{i}\right\}, p\right)}\left(\left|\alpha_{\mathrm{sp}(\Gamma)}\right|>a^{*}|\Gamma|\right)+Z_{\lambda, \mathrm{sp}(\Gamma)}^{\left(\left\{\epsilon_{i}\right\}, p\right)}\left(\left|\alpha_{\mathrm{sp}(\Gamma)}\right| \leq a^{*}|\Gamma|\right)
$$

and use (4.9) to get (4.10).

The proof of (4.1) for the fat contours is not as simple as for the slim ones. An important point, see Proposition 4.4 below, is the analysis of the empirical averages of $\alpha_{x}$ 
in regions where only slim contours are present and where the boundary conditions are in the minus phase. The setup is described by the measures

$$
\mu_{\lambda, \Lambda}^{(-, p, s)}(d q)=\mu_{\lambda, \Lambda}^{(-, p)}(d q \mid\{\text { all contours are slim }\})
$$

To estimate the contribution of the perturbation $\lambda H_{I}$, we will prove that $\alpha_{\Lambda}$ is close to $\alpha_{-}|\Lambda|$, which is not a priori obvious because $\lambda>0$. After establishing a Peierls bound on contours for the measure $\mu_{\lambda, \Lambda}^{(-, p, s)}$, we will conclude that most of the space is made of sites $x$ with $\Theta(\cdot ; x)=-1$ and this will yield the desired result.

Lemma 4.3. Let $\Lambda$ be a fat region and $\Delta \sqsubset \Lambda,|\Delta|<\xi_{-}(\lambda)^{d}$. Then for $\epsilon_{0}= \pm 1$,

$$
\mu_{\lambda, \Lambda}^{(-, p, s)}\left(d q \mid \mathcal{F}_{\Delta^{c}},\left\{\Theta(q ; x)=\epsilon_{0}, \operatorname{dist}(x, \Delta)=1\right\}\right)=\mu_{\lambda, \Delta}^{\left(\epsilon_{0}, p\right)}(d q)
$$

As a consequence if $\Gamma$ is a slim contour with $\operatorname{sp}(\Gamma) \sqsubset \Lambda$, then

$$
\mu_{\lambda, \Lambda}^{(-, p, s)}(\Gamma ; p)=m_{\lambda}(\Gamma ; p)
$$

the r.h.s. having been defined in (3.2).

Proof. The measure on the l.h.s. of (4.12) is equal to

$$
\mu_{\lambda, \Lambda}^{(-, p)}\left(d q \mid \mathcal{F}_{\Delta^{c}},\left\{\Theta(q ; x)=\epsilon_{0}, \operatorname{dist}(x, \Delta)=1\right\},\{\text { all contours of } q \text { are slim }\}\right)\left(q^{\prime}\right)
$$

Since $\Theta(q ; x)=\epsilon_{0}$ for all $x: \operatorname{dist}(x, \Delta)=1$, the contours of $q$ have spatial support either contained entirely in $\Delta$ or entirely outside $\Delta$. Since $|\Delta|<\xi_{-}(\lambda)^{d}$, we can drop the condition that the contours whose spatial support is in $\Delta$ are slim, because it is automatically satisfied, hence (4.12).

If $\Gamma$ is a slim contour and $\operatorname{sp}(\Gamma) \sqsubset \Lambda$, then $V(\Gamma) \sqsubset \Lambda$ because $\Lambda$ is fat and (4.13) follows then from (4.12). Lemma 4.3 is proved.

Proposition 4.4. There is $c>0$ so that if $0<\lambda \leq \lambda^{*}$ and $\Lambda$ is a fat region, then

$$
\begin{gathered}
\mu_{\lambda, \Lambda}^{(-, p, s)}\left(q:\left|\alpha_{\Lambda}(q)-\alpha_{-}\right| \Lambda||>2 \zeta|\Lambda|\right) \leq c e^{-C_{P} / 4} \\
\mu_{0, \Lambda}^{(+, p)}\left(q:\left|\alpha_{\Lambda}(q)-\alpha_{+}\right| \Lambda||>2 \zeta|\Lambda|\right) \leq c e^{-C_{P} / 4}
\end{gathered}
$$

Proof. We start with (4.15). Since

$$
\left\{\left|\alpha_{\Lambda}-\alpha_{+}\right| \Lambda||>2 \zeta|\Lambda|\right\} \sqsubset\left\{\sum_{x \in \Lambda}\left|\alpha_{x}-\alpha_{+}\right| \mathbf{1}_{\left|\alpha_{x}-\alpha_{+}\right|>\zeta}>\zeta|\Lambda|\right\}
$$

by the Chebishev inequality,

$$
\mu_{0, \Lambda}^{(+, p)}\left(q:\left|\alpha_{\Lambda}(q)-\alpha_{+}\right| \Lambda||>2 \zeta|\Lambda|\right) \leq \frac{1}{\zeta} \sup _{x \in \Lambda} \mu_{0, \Lambda}^{(+, p)}\left(\left|\alpha_{x}-\alpha_{+}\right| \mathbf{1}_{\left|\alpha_{x}-\alpha_{+}\right|>\zeta}\right)
$$


All $q$ in the support of $\mu_{0, \Lambda}^{(+, p)}$ are such that

$$
\text { if }\left|\alpha_{x}(q)-\alpha_{+}\right|>\zeta \text { then } q \text { has a contour } \Gamma \text { with } x \in V(\Gamma)
$$

Then, supposing $C_{P}$ large enough, by Lemma 3.2

$$
\mu_{0, \Lambda}^{(+, p)}\left(q:\left|\alpha_{\Lambda}(q)-\alpha_{+}\right| \Lambda||>2 \zeta|\Lambda|\right) \leq \frac{\left(e^{-C_{P}}\right)^{1 / 2}}{\zeta} \sup _{x \in \Lambda} \mu_{0, \Lambda}^{(+, p)}\left(\left|\alpha_{x}-\alpha_{+}\right|^{2}\right)^{1 / 2}
$$

(4.15) follows then from (4.19) by using (2.11).

Analogously we have

$$
\mu_{\lambda, \Lambda}^{(-, p, s)}\left(q:\left|\alpha_{\Lambda}(q)-\alpha_{-}\right| \Lambda||>2 \zeta|\Lambda|\right) \leq \frac{1}{\zeta} \sup _{x \in \Lambda} \mu_{\lambda, \Lambda}^{(-, p, s)}\left(\left|\alpha_{x}-\alpha_{-}\right| \mathbf{1}_{\left|\alpha_{x}-\alpha_{-}\right|>\zeta}\right)
$$

For any $x$ on the r.h.s. of (4.20) there is a slim contour $\Gamma$ such that $V(\Gamma) \ni x$. Then

$$
\mu_{\lambda, \Lambda}^{(-, p, s)}\left(\left|\alpha_{x}-\alpha_{-}\right| \mathbf{1}_{\left|\alpha_{x}-\alpha_{-}\right|>\zeta}\right) \leq \sum_{\substack{\Gamma \text { is slim } \\ V(\Gamma) \ni x}} \mu_{\lambda, \Lambda}^{(-, p, s)}\left(\left|\alpha_{x}-\alpha_{-}\right| \mathbf{1}_{q \rightarrow \Gamma}\right)
$$

where $q \rightarrow \Gamma$ means that $\Gamma$ is a contour for $q$. By (4.12) we get

$$
\mu_{\lambda, \Lambda}^{(-, p, s)}\left(\left|\alpha_{x}-\alpha_{-}\right| \mathbf{1}_{q \rightarrow \Gamma}\right)=\int \mu_{\lambda, \Lambda}^{(-, p, s)}(d q) \mu_{\lambda, V(\Gamma)}^{(\epsilon(\Gamma), q)}\left(\left|\alpha_{x}\left(q^{\prime}\right)-\alpha_{-}\right| \mathbf{1}_{q^{\prime} \rightarrow \Gamma}\right)
$$

Using (4.4), setting $\epsilon(\Gamma)= \pm 1$ if $\Gamma$ is a \pm contour, we then get

$$
\mu_{\lambda, V(\Gamma)}^{(\epsilon(\Gamma), q)}\left(\left|\alpha_{x}\left(q^{\prime}\right)-\alpha_{-}\right| \mathbf{1}_{q^{\prime} \rightarrow \Gamma}\right) \leq e^{-\left(C_{P} / 4\right)|\Gamma|} \mu_{\lambda, V(\Gamma)}^{(\epsilon(\Gamma), q)}\left(\left|\alpha_{x}-\alpha_{-}\right|\right)^{1 / 2}
$$

Using again (2.11), we conclude that if $C_{P}$ is large enough, then (4.14) holds. Proposition 4.4 is proved.

The minus layer attached to a plus contour

Resuming the notation (4.5), we fix a fat + contour $\Gamma_{0},\left(\Gamma_{0}\right.$ because we will use $\Gamma$ as a current symbol for contours, + because we are proving (4.1) with $\lambda>0$ ). We also suppose that $\operatorname{int}^{-}\left(\Gamma_{0}\right)$ is a fat region, in the other case the proof of (4.1) is essentially the same as that of Proposition 4.2 and omitted. Given a configuration $q$ which has $\Gamma_{0}$ as a contour, we select inside $\operatorname{int}^{-}\left(\Gamma_{0}\right)$ the family $\left(\Gamma_{1}, . ., \Gamma_{n}\right)$ of the fat, minus, "external" contours: these are all the fat, minus contours $\Gamma$ with $\operatorname{sp}(\Gamma) \sqsubset \operatorname{int}^{-}\left(\Gamma_{0}\right)$ and which are neither contained in any $V\left(\Gamma^{\prime}\right), \Gamma^{\prime}$ a plus contour in $\operatorname{int}^{-}\left(\Gamma_{0}\right)$, nor in the plus interior of any minus contour $\Gamma^{\prime}$ in $\operatorname{int}^{-}\left(\Gamma_{0}\right)$. Thus $\left(\Gamma_{1}, . ., \Gamma_{n}\right)$ are all the fat contours in the subset of $\operatorname{int}^{-}\left(\Gamma_{0}\right)$ obtained by erasing from the latter all the regions which are surrounded by a circuit with $\Theta=1$. We will shorthand $\underline{\Gamma}=\left(\Gamma_{0}, \Gamma_{1}, . ., \Gamma_{n}\right)$, with $\left(\Gamma_{1}, . ., \Gamma_{n}\right)$ as above.

Thus $\underline{\Gamma}$ divides $V\left(\Gamma_{0}\right)$ into three regions:

$$
\operatorname{int}^{+}(\underline{\Gamma})=\bigsqcup_{i} \operatorname{int}^{+}\left(\Gamma_{i}\right), \quad \operatorname{sp}(\underline{\Gamma})=\bigsqcup_{i} \operatorname{sp}\left(\Gamma_{i}\right), \quad \Delta^{-}(\underline{\Gamma}):=V\left(\Gamma_{0}\right) \backslash\left\{\operatorname{int}^{+}(\underline{\Gamma}) \sqcup \operatorname{sp}(\underline{\Gamma})\right\}
$$


The minus layer attached to the plus contour $\Gamma_{0}$ is then defined as

$$
\Lambda^{-}(\underline{\Gamma})=V\left(\Gamma_{0}\right) \backslash \operatorname{int}^{+}(\underline{\Gamma})=\operatorname{sp}(\underline{\Gamma}) \sqcup \Delta^{-}(\underline{\Gamma})
$$

In the next theorem we will prove a bound on the volume of the minus layer $\Lambda^{-}(\underline{\Gamma})$ which implies (4.1) and which will be crucial in the proof in the next section that all tempered DLR measures are in $\mathcal{G}_{\lambda}^{+}$, if $\lambda>0$ (and in $\mathcal{G}_{\lambda}^{-}$, if $\lambda<0$ ).

Theorem 4.5. Let $\Gamma_{0}$ be a fat, plus contour and $\Lambda^{-}$the random set defined in (4.22), then for any $V \geq\left|\Gamma_{0}\right|$,

$$
\mu_{\lambda, V\left(\Gamma_{0}\right)}^{(+, p)}\left(\exists \Gamma_{0} \wedge\left|\Lambda^{-}\right| \geq V\right) \leq e^{-\left(C_{P} / 8\right)\left|\Gamma_{0}\right|-\omega V}, \quad \omega=\min \left\{\frac{C_{P}}{8} ; \lambda\left(\beta \ell^{d}\right) \frac{\alpha_{+}-\alpha_{-}}{2}\right\}
$$

As a consequence

$$
m_{\lambda}\left(\Gamma_{0} ; p\right) \leq e^{-\left(C_{P} / 8\right)\left|\Gamma_{0}\right|}
$$

Proof. Note first that (4.24) is an obvious consequence of (4.23). Thus we only need to prove the latter inequality.

With the obvious meaning of the symbols, we have

$$
\mu_{\lambda, V\left(\Gamma_{0}\right)}^{(+, p)}\left(\left|\Lambda^{-}\right| \geq V\right)=\frac{Z_{\lambda, V\left(\Gamma_{0}\right)}^{(+, p)}\left(\left|\Lambda^{-}\right| \geq V\right)}{Z_{\lambda, V\left(\Gamma_{0}\right)}^{(+, p)}}
$$

To bound the partition function in the numerator we start writing

$$
\begin{gathered}
Z_{\lambda, V\left(\Gamma_{0}\right)}^{(+, p)}\left(\left|\Lambda^{-}\right| \geq V\right) \leq \sum_{\underline{\Gamma}:\left|\Lambda^{-}\right| \geq V} Z_{\lambda, V\left(\Gamma_{0}\right)}^{(+, p)}(q \rightarrow \underline{\Gamma}) \\
Z_{\lambda, V\left(\Gamma_{0}\right)}^{(+, p)}(q \rightarrow \underline{\Gamma})=\int_{\left\{p^{\prime} \rightarrow \text { int }^{+}(\underline{\Gamma})\right\}} \nu_{\text {int }^{+}(\underline{\Gamma})}\left(d p^{\prime}\right) e^{-H_{\lambda}\left(p^{\prime}\right)} Z_{\lambda, \Lambda^{-}}^{\left(+, p, p^{\prime}\right)}(q \rightarrow \underline{\Gamma})
\end{gathered}
$$

where $\left\{p^{\prime} \rightarrow \operatorname{int}^{+}(\underline{\Gamma})\right\}$ is the condition

$$
\theta\left(p_{x}^{\prime}\right)=1 \text { for all } x \in \operatorname{int}^{+}(\underline{\Gamma}): \operatorname{dist}\left(x, \Lambda^{-}\right) \leq 2
$$

while $\{q \rightarrow \underline{\Gamma}\}$ in the last partition function is a shorthand for the condition that the configuration equal to $q$ in $\Lambda^{-}$, to $p^{\prime}$ in $\operatorname{int}^{+}(\underline{\Gamma})$ and to $p$ outside $V\left(\Gamma_{0}\right)$ gives rise to $\underline{\Gamma}$.

The rest of the proof will only involve manipulations on $Z_{\lambda, \Lambda^{-}}^{\left(+, p, p^{\prime}\right)}(q \rightarrow \underline{\Gamma})$. Calling

$$
\Delta_{0}^{-}=\left\{x \in \Delta^{-}: \operatorname{dist}\left(x,\left(\Delta^{-}\right)^{c}\right)>1\right\}, \quad \operatorname{sp}(\underline{\Gamma})^{+}=\Lambda^{-} \backslash \Delta_{0}^{-}
$$

(recall that $\operatorname{sp}(\underline{\Gamma})=\Lambda^{-} \backslash \Delta^{-}$), we then have

$$
Z_{\lambda, \Lambda^{-}}^{\left(+, p, p^{\prime}\right)}(q \rightarrow \underline{\Gamma})=\int_{\left\{\theta\left(q_{x}^{\prime}\right)=\theta_{\underline{\Gamma}}\right\}} \nu_{\mathrm{sp}(\underline{\Gamma})^{+}}\left(d q^{\prime}\right) e^{-H_{\lambda}\left(q^{\prime} \mid p, p^{\prime}\right)} Z_{\lambda, \Delta_{0}^{-}}^{\left(-, q^{\prime}, s\right)}
$$


where $\left\{\theta\left(q_{x}^{\prime}\right)=\theta_{\underline{\Gamma}}\right\}$ is a shorthand for the condition that $\theta\left(q_{x}^{\prime}\right)=\theta_{\Gamma_{i}}(x)$ when $x \in \operatorname{sp}\left(\Gamma_{i}\right)$, $i=0, . ., n$, and moreover that $\theta\left(q_{x}^{\prime}\right)=-1$ when $x \in \operatorname{sp}(\underline{\Gamma})^{+} \backslash \operatorname{sp}(\underline{\Gamma})$ (which is the same as $\Delta^{-} \backslash \Delta_{0}^{-}$.

By (4.14) with $\Lambda \rightarrow \Delta_{0}^{-}$,

$$
Z_{\lambda, \Delta_{0}^{-}}^{\left(-, q^{\prime}, s\right)} \leq\left(1-c e^{-C_{P} / 4}\right)^{-1} Z_{\lambda, \Delta_{0}^{-}}^{\left(-, q^{\prime}, s\right)}\left(\left|\alpha_{\Delta_{0}^{-}}-\alpha_{-}\right| \Delta_{0}^{-}|| \leq 2 \zeta\left|\Delta_{0}^{-}\right|\right)
$$

which, reinserted into (4.25) yields:

$$
\begin{aligned}
Z_{\lambda, \Lambda^{-}}^{\left(+, p, p^{\prime}\right)}(q \rightarrow \underline{\Gamma}) & =\int_{\left|\alpha_{\Delta_{0}^{-}}-\alpha_{-}\right| \Delta_{0}^{-} \| \leq 2 \zeta\left|\Delta_{0}^{-}\right| ; \theta\left(q_{x}\right)=-1, x \in \Delta^{-} \backslash \Delta_{0}^{-}} \nu_{\Delta^{-}}\left(d q^{\prime}\right) e^{-H_{\lambda}\left(q^{\prime} \mid p, p^{\prime}\right)} \\
& \times \prod_{i=0}^{n} \int_{\theta\left(q_{x}\right)=\theta_{\Gamma_{i}}(x), x \in \operatorname{sp}\left(\Gamma_{i}\right)} \nu_{\mathrm{sp}\left(\underline{\Gamma}_{i}\right)}(d q) e^{-H_{\lambda}\left(q \mid q^{\prime}, p \cdot p^{\prime}\right)}
\end{aligned}
$$

In each one of the integrals in the above product we distinguish whether $\left|\alpha_{\mathrm{sp}\left(\Gamma_{j}\right)}\right|>a^{*}\left|\Gamma_{j}\right|$ or else the reverse inequality holds. When the former occurs, we use (4.10) and write

$$
\begin{array}{r}
\int_{\theta\left(q_{x}\right)=\theta_{\Gamma_{j}}(x), x \in \operatorname{sp}\left(\Gamma_{j}\right)} \nu_{\mathrm{sp}\left(\Gamma_{j}\right)}(d q) e^{-H_{\lambda}\left(q \mid q^{\prime}, p, p^{\prime}\right)} \mathbf{1}_{\left|\alpha_{\mathrm{sp}\left(\Gamma_{j}\right)}\right|>a^{*}\left|\Gamma_{j}\right|} \\
\leq e^{-C_{P}\left|\Gamma_{j}\right|+1} Z_{\lambda, \mathrm{sp}\left(\Gamma_{j}\right)}^{\left(\left\{\epsilon_{i}\right\}_{j}, q^{\prime}, p, p^{\prime}\right)}\left(\left|\alpha_{\mathrm{sp}\left(\Gamma_{j}\right)}\right| \leq a^{*}\left|\Gamma_{j}\right|\right)
\end{array}
$$

where $\left\{\epsilon_{i}\right\}_{j}$ are the values of $\theta$ on the boundaries of $\operatorname{sp}\left(\Gamma_{j}\right)$, which are fixed by $\underline{\Gamma}$.

Collecting the above inequalities and calling $\left|\Gamma_{I^{c}}\right|, I \sqsubset\{0, \ldots, n\}$, the sum of $\left|\Gamma_{j}\right|$ over $j \in\{0, . ., n\} \backslash I$, we get from $(4.26)$

$$
\begin{aligned}
& Z_{\lambda, \Lambda^{-}}^{\left(+, p, p^{\prime}\right)}(q \rightarrow \underline{\Gamma}) \leq \sum_{I \sqsubset\{0, . ., n\}} e^{-C_{P}\left|\Gamma_{I^{c}}\right|+n+1} \\
& \times Z_{\lambda, \Lambda^{-}}^{\left(+, p, p^{\prime}\right)}\left(\left\{\left|\frac{\alpha_{\Delta^{-}}}{\left|\Delta^{-}\right|}-\alpha_{-}\right| \leq 2 \zeta\right\},\left\{\left|\frac{\alpha_{\mathrm{sp} \Gamma_{i}}}{\left|\Gamma_{i}\right|}\right| \leq a^{*}, \forall i\right\},\left\{q \rightarrow \Gamma_{i}, i \in I\right\}\right)
\end{aligned}
$$

having used that $\left\{\theta=-1\right.$ on $\left.\Delta^{-} \backslash \Delta_{0}^{-}\right\}$implies that

$$
\left|\alpha_{\Delta^{-} \backslash \Delta_{0}^{-}}-\alpha_{-}\right| \Delta^{-} \backslash \Delta_{0}^{-}|| \leq \zeta\left|\Delta^{-} \backslash \Delta_{0}^{-}\right|
$$

We next change $\lambda$ into $\lambda=0$ by exploiting the constraints in the last partition function:

$$
\begin{aligned}
Z_{\lambda, \Lambda^{-}}^{\left(+, p, p^{\prime}\right)}(q \rightarrow \underline{\Gamma}) \leq & \sum_{I \sqsubset\{0, . ., n\}} e^{-C_{P}\left|\Gamma_{I^{c}}\right|+\lambda\left(\beta \ell^{d}\right)\left(\alpha_{-}+2 \zeta\right)\left|\Lambda^{-}\right|} e^{n+1+\lambda\left(\beta \ell^{d}\right)\left[-\left(\alpha_{-}+2 \zeta\right)+a^{*}\right]|\underline{\Gamma}|} \\
& \times Z_{0, \Lambda^{-}}^{\left(+, p, p^{\prime}\right)}\left(\left\{q \rightarrow \Gamma_{i}, i \in I\right\}\right)
\end{aligned}
$$

and use the Peierls bound

$$
Z_{0, \Lambda^{-}}^{\left(+, p, p^{\prime}\right)}\left(\left\{q \rightarrow \Gamma_{i}, i \in I\right\}\right) \leq e^{-C_{P}\left|\Gamma_{I}\right|} Z_{0, \Lambda^{-}}^{\left(+, p, p^{\prime}\right)}
$$


We will next reconstruct a partition function with the correct value of $\lambda$ and then conclude the proof of the theorem. By (4.15),

$$
Z_{0, \Lambda^{-}}^{\left(+, p, p^{\prime}\right)} \leq\left(1+c e^{-C_{P} / 4}\right) Z_{0, \Lambda^{-}}^{\left(+, p, p^{\prime}\right)}\left(\left|\alpha_{\Lambda^{-}}-\alpha_{+}\right| \Lambda^{-}|| \leq 2 \zeta\left|\Lambda^{-}\right|\right)
$$

Supposing $C_{P}$ so large that $\left(1+c e^{-C_{P} / 4}\right) \leq e$,

$$
Z_{0, \Lambda^{-}}^{\left(+, p, p^{\prime}\right)} \leq e^{1-\lambda\left(\beta \ell^{d}\right)\left(\alpha_{+}-2 \zeta\right)\left|\Lambda^{-}\right|} Z_{\lambda, \Lambda^{-}}^{\left(+, p, p^{\prime}\right)}
$$

By the two equations above, (4.27) becomes

$$
\begin{gathered}
Z_{\lambda, \Lambda^{-}}^{\left(+, p, p^{\prime}\right)}(q \rightarrow \underline{\Gamma}) \leq 2^{n+1} e^{-C_{P}|\underline{\Gamma}|-\lambda\left(\beta \ell^{d}\right)\left(\alpha_{+}-\alpha_{-}-4 \zeta\right)\left|\Lambda^{-}\right|} e^{n+2+\lambda\left(\beta \ell^{d}\right)\left[-\left(\alpha_{-}+2 \zeta\right)+a^{*}\right]|\underline{\mid}|} \\
\times Z_{\lambda, \Lambda^{-}}^{\left(+, p, p^{\prime}\right)}
\end{gathered}
$$

with $2^{n+1}$ counting the cardinality of the subsets $I$ in (4.27). By the choice of $\zeta$, see (2.5),

$$
\alpha_{+}-\alpha_{-}-4 \zeta=\frac{\alpha_{+}-\alpha_{-}}{2}+\frac{\alpha_{+}-\alpha_{-}}{10}
$$

and by choosing properly the constant $C(d)$ in Theorem 2.1,

$$
\left\{-\frac{7 C_{P}}{8}+\delta^{-1} C_{P} \lambda\left[-\alpha_{-}+a^{*}\right]\right\}|\underline{\Gamma}|+(n+2)+(n+1) \log 2 \leq-\frac{C_{P}}{4}|\underline{\Gamma}|
$$

we finally get:

$$
\frac{Z_{\lambda, V\left(\Gamma_{0}\right)}^{(+, p)}\left(\left|\Lambda^{-}\right| \geq V\right)}{Z_{\lambda, V\left(\Gamma_{0}\right)}^{(+, p)}} \leq e^{-C_{P}\left|\Gamma_{0}\right| / 8-\omega V} \sum_{\underline{\Gamma}:\left|\Lambda^{-}\right| \geq V} e^{-C_{P}|\underline{\Gamma}| / 4-\lambda\left(\beta \ell^{d}\right)\left(\alpha_{+}-\alpha_{-}\right)\left|\Lambda^{-}\right| / 10}
$$

We will prove that

$$
\hat{m}_{\lambda}\left(\Gamma_{0} ; p\right):=\sum_{\underline{\Gamma}} e^{-C_{P}|\underline{\Gamma}| / 4-\lambda\left(\beta \ell^{d}\right)\left(\alpha_{+}-\alpha_{-}\right)\left|\Lambda^{-}\right| / 10} \leq 1
$$

which will conclude the proof of the theorem.

\section{A counting argument}

The proof of (4.30) will use coarse graining with grains the cubes of size $\xi_{+}(\lambda)$ of a partition $\mathcal{D}^{\left(\xi_{+}(\lambda)\right)}$ of $\mathbb{Z}^{d}$, where

$$
\xi_{+}(\lambda)=\xi_{-}(\lambda)^{10}
$$

supposing for simplicity $\xi_{+}(\lambda) \in \mathbb{N}$.

Call $\left\{\hat{C}_{x}, x \in X\right\}, X$ a label set, the cubes of $\mathcal{D}^{\left(\xi_{+}(\lambda)\right)}$ with non empty intersection with $V\left(\Gamma_{0}\right)$, in the sequel we will restrict to such a set. Given $\underline{\Gamma}$, each $\hat{C}_{x}, x \in X$, falls into one of the following three categories:

$$
\text { - } \hat{C}_{x} \sqsubset \Delta^{-}(\underline{\Gamma}), \quad \text { - } \hat{C}_{x} \sqcap \operatorname{sp}(\underline{\Gamma}) \neq \emptyset, \quad \text { • } \hat{C}_{x} \sqsubset \operatorname{int}^{+}(\underline{\Gamma})
$$

In fact the fourth one, namely $\hat{C}_{x} \sqcap \Delta^{-}(\underline{\Gamma}) \neq \emptyset, \hat{C}_{x} \sqcap \operatorname{int}^{+}(\underline{\Gamma}) \neq \emptyset$ and $\hat{C}_{x} \sqcap \operatorname{sp}(\underline{\Gamma})=\emptyset$ is empty because of the definition of contours. 
To distinguish the three cases we introduce the variable $\psi(\underline{\Gamma} ; x), x \in X$, which has value $-1,0$ and 1 respectively in the first, second and third case above. For ease of reference we write it down explicitly:

$$
\psi(\underline{\Gamma} ; x)= \begin{cases}-1 & \text { if } \hat{C}_{x} \sqsubset \Delta^{-}(\underline{\Gamma}) \\ 0 & \text { if } \hat{C}_{x} \sqcap \operatorname{sp}(\underline{\Gamma}) \neq \emptyset \\ 1 & \text { if } \hat{C}_{x} \sqsubset \operatorname{int}^{+}(\underline{\Gamma})\end{cases}
$$

For each $x \in X$ we introduce a weight $w_{\lambda}(\underline{\Gamma} ; x)$ which is determined by the value of $\psi(\underline{\Gamma} ; x)$. We first call, for $x \in X$,

$$
\hat{C}_{x}^{+}=\left\{y: \operatorname{dist}\left(y, \hat{C}_{x}\right) \leq \xi_{+}(\lambda)\right\}, \quad \underline{\Gamma}_{x}=\left\{\Gamma_{i} \in \underline{\Gamma}: \operatorname{sp}\left(\Gamma_{i}\right) \sqcap \hat{C}_{x} \neq \emptyset\right\}
$$

and then define the weights

$$
w_{\lambda}(\underline{\Gamma} ; x)= \begin{cases}e^{-\lambda\left(\beta \ell^{d}\right)\left[\left(\alpha_{+}-\alpha_{-}\right) / 10\right] \xi_{+}(\lambda)^{d}} & \text { if } \psi(\underline{\Gamma} ; x)=-1 \\ e^{-3^{-d}\left(C_{P} / 4\right)\left|\operatorname{sp}\left(\underline{\Gamma}_{x}\right) \sqcap C_{x}^{+}\right|} & \text {if } \psi(\underline{\Gamma} ; x)=0 \\ 1 & \text { if } \psi(\underline{\Gamma} ; x)=1\end{cases}
$$

The definition is such that

$$
\hat{m}_{\lambda}\left(\Gamma_{0} ; p\right) \leq \sum_{\underline{\Gamma}} \prod_{x \in X} w_{\lambda}(\underline{\Gamma} ; x)
$$

The difficulty with (4.35) is that the weights $w_{\lambda}(\cdot ; x)$ for different $x$ are not independent, but, as we will see, we can reduce by upper bounds to the independent case. Let

$$
S=\left\{s \in\{0, \pm 1\}^{X}: s_{x}=0 \text { for } \hat{C}_{x} \sqcap \operatorname{sp}\left(\Gamma_{0}\right) \neq \emptyset, \bigsqcup_{s_{x}<1} \hat{C}_{x} \text { is } \star \text { connected }\right\}
$$

Our goal is to define new weights $\omega_{\lambda}\left(s_{x}\right), x \in X$, so that

$$
\hat{m}_{\lambda}\left(\Gamma_{0} ; p\right) \leq \sum_{s \in S} \prod_{x \in X} \omega_{\lambda}\left(s_{x}\right)
$$

Recalling (4.34) we set $\omega_{\lambda}(1)=1$ and

$$
\omega_{\lambda}(-1)=e^{-\lambda\left(\beta \ell^{d}\right)\left[\left(\alpha_{+}-\alpha_{-}\right) / 10\right] \xi_{+}(\lambda)^{d}}
$$

To define $\omega_{\lambda}(0)$, we denote by $\underline{T}=\left(T_{1}, . ., T_{n}\right)$ any finite sequence of disjoint, $\star$ connected, $\mathcal{D}^{(1)}$-measurable sets and define, given a cube $\hat{C} \in \mathcal{D}^{\left(\xi_{+}(\lambda)\right)}$,

$$
\omega_{\lambda}(0)=\sum_{\substack{T: T_{i} \sqcap \hat{C} \neq \emptyset \\\left|T_{i}\right| \geq N^{*}}} \prod_{i} 3^{\left|T_{i}\right|} e^{-3^{-d}\left(C_{P} / 4\right)\left|T_{i}\right|}
$$

where

$$
N^{*}=\min \left\{c_{d} \xi_{-}(\lambda)^{d-1} ; \xi_{+}(\lambda)\right\}
$$


$c_{d}$ an isoperimetric constant whose value will be specified later. We will prove (4.37) by showing that

$$
\sum_{\underline{\Gamma}: \psi(\underline{\Gamma} ; x)=s_{x}, x \in X} \prod_{x \in X} w_{\lambda}(\underline{\Gamma} ; x) \leq \prod_{x \in X} \omega_{\lambda}\left(s_{x}\right)
$$

Indeed the factors $3^{\left|T_{i}\right|}$ in (4.39) take into account that there at most $3^{\left|T_{i}\right|}$ contours $\Gamma$ with $\operatorname{sp}(\Gamma)=T_{i} . N^{*}$ is a lower bound for the number of sites of all sets $\operatorname{sp}(\Gamma)$ which are in $\hat{C}_{x}^{+}$, over all fat contours $\Gamma$ such that $\operatorname{sp}(\Gamma) \sqcap \hat{C}_{x} \neq \emptyset$ : the first term is a lower bound for $|\Gamma|$ and covers the case when $\operatorname{sp}(\Gamma) \sqsubset \hat{C}_{x}^{+}$(see (4.7) and (4.3)), the second one is for the case when $\operatorname{sp}(\Gamma)$ is not contained in $\hat{C}_{x}^{+}$. With this choice (4.41) holds.

We next need to bound the weights $\omega(\cdot)$. For $C_{0}$ in (4.2) large enough, $N^{*}$ becomes large too, and if $C_{P}$ is also large enough

$$
\begin{aligned}
\omega_{\lambda}(0) & \leq e^{-3^{-d}\left(C_{P} / 8\right) N^{*}}\left(1+\sum_{T \ni 0,|T| \geq N^{*}} 3^{|T|} e^{-3^{-d}\left(C_{P} / 8\right)|T|}\right)^{\left[\xi_{+}(\lambda)\right]^{d}} \\
& \leq 2 e^{-3^{-d}\left(C_{P} / 8\right) N^{*}}
\end{aligned}
$$

The proof of (4.42) uses standard combinatorics arguments and it is omitted.

Recalling (4.34),(4.31) and (4.2)

$$
\omega_{\lambda}(-1)=e^{-\lambda\left(\beta \ell^{d}\right)\left[\left(\alpha_{+}-\alpha_{-}\right) / 10\right] \xi_{+}(\lambda)^{d}}, \quad \lambda \xi_{+}(\lambda)^{d}=\lambda^{1-5 d}
$$

Thus by choosing $\lambda^{*}$ small enough, also $\omega_{\lambda}(-1)$ can be made as small as desired. By standard arguments, which again are omitted, it is then possible to show that $\hat{m}_{\lambda}\left(\Gamma_{0} ; p\right)<1$ and (4.1) is proved.

\section{Uniqueness of phases}

In this section we will prove that when $0<\lambda \leq \lambda^{*}$ any tempered DLR measure $\mu$ is in $\mathcal{G}_{\lambda}^{+}$, the proof of the analogous statement for $\lambda<0$ is omitted. The idea of the proof is similar to that in $[\mathbf{8}]$ and $[\mathbf{1}]$.

Let $\Lambda_{0}$ be a cube of side $L$ and $\Lambda^{\prime}$ the cube with same center and side $L / 2$. Given a configuration $q$, let $G=G(q)$ be the union of the maximal connected components of $\{x: \Theta(q ; x)<1\}$ which have intersection with $\Lambda_{0}^{c}$. Call

$$
G^{+}=\{x: \operatorname{dist}(x, G) \leq 1\}, \quad \Delta=\left(G^{+}\right)^{c} \sqcap \Lambda_{0}
$$


Given any bounded, local function $f$, we consider below $L$ so large that $f=f\left(q_{\Lambda^{\prime}}\right)$ (i.e. it only depends on $\left.q_{\Lambda^{\prime}}\right)$. We then have

$$
\int \mu(d q) f(q)=\sum_{\Delta \not \supset \Lambda^{\prime}} \int \mu(d q) f(q) \mathbf{1}_{\Delta(q)=\Delta}+\sum_{\Delta \sqsupset \Lambda^{\prime}} \int \mu(d p) \int \mu_{\lambda, \Delta}^{(+, p)}\left(d q_{\Lambda^{\prime}}\right) f\left(q_{\Lambda^{\prime}}\right) \mathbf{1}_{\Delta(p)=\Delta}
$$

Recalling that the dependence of $\Lambda, \Delta, \Lambda^{\prime}$ on $L$ is not made explicit, we will prove

$$
\lim _{L \rightarrow \infty} \mu\left(\Delta \not \supset \Lambda^{\prime}\right)=0
$$

thus concluding that

$$
\begin{aligned}
& \int \mu(d q) f(q)=\lim _{L \rightarrow \infty} \sum_{\Delta \sqsupset \Lambda^{\prime}} \int m_{L}(d p ; \Delta) \int \mu_{\lambda, \Delta}^{(+, p)}\left(d q_{\Lambda^{\prime}}\right) f\left(q_{\Lambda^{\prime}}\right) \\
& m_{L}(d p ; \Delta)=\left(\frac{\mathbf{1}_{\Delta(p)=\Delta}}{\mu\left(\Delta \sqsupset \Lambda^{\prime}\right)}\right) \mu(d p), \quad \sum_{\Delta \sqsupset \Lambda^{\prime}} \int m_{L}(d p ; \Delta)=1
\end{aligned}
$$

For any $L$ the r.h.s. of $(5.3)$ is the expectation of $f$ w.r.t. a measure in $\mathcal{G}_{\lambda, \Lambda^{\prime}}^{+}$, hence, by the arbitrariness of $f, \mu \in \mathcal{G}_{\lambda}^{+}$.

In the remaining part of the section we will prove (5.2).

\section{The random sets $G_{k}^{-}$.}

It is convenient, at this point, to use the function $\theta\left(q_{x}\right)$ rather than the phase indicator $\Theta(q ; x)$, due to the local structure of the former. Recalling $(2.2)$,

$$
\{\Theta<1\}=\{x: \operatorname{dist}(x,\{\theta<1\}) \leq 1\}
$$

so that the maximal connected components of $\{\Theta<1\}$ are obtained from the maximal 3connected components of $\{\theta<1\}$ (two sites $x$ and $y$ being called 3-connected if $|x-y| \leq 3$ ) by taking all sites which have distance $\leq 1$ from the latter. As a consequence, defining $G^{-}=G^{-}(q)=\left\{x \in G: \theta\left(q_{x}\right)<1\right\}$, we recover $G$ from $G^{-}$by setting $G=\{x$ : $\left.\operatorname{dist}\left(x, G^{-}\right) \leq 1\right\}$; moreover, the part of $G^{-}$contained in a maximal connected component of $G$ is a 3-connected set and distinct 3-connected components of $G^{-}$belong to distinct connected components of $G$. $G^{-}$can be defined directly as the union of all the maximal 3-connected components of $\left\{x: \theta\left(q_{x}\right)<1\right\}$ which have distance $\leq 1$ from $\Lambda_{0}^{c}$.

We will next approximate the set $G^{-}$by sets $G_{k}^{-}$. For $k \geq 1$, let

$$
\Lambda_{k}=\Lambda_{k-1} \backslash\left\{x: \operatorname{dist}\left(x, \Lambda_{k-1}^{c}\right) \leq 3\right\}
$$

and define $G_{k}^{-}$as equal to the union of all the maximal 3-connected components of $\{x \in$ $\left.\Lambda_{k}^{c}: \theta\left(q_{x}\right)<1\right\}$ which have distance $\leq 1$ from $\Lambda_{0}^{c}$. We call $M_{k}^{-}=G_{k}^{-} \sqcap\left(\Lambda_{k-1} \backslash \Lambda_{k}\right)$. Since $M_{k}^{-}=\emptyset$ implies that $\theta\left(q_{x}\right)=1$ for all $1 \leq \operatorname{dist}\left(x, G_{K}^{-}\right) \leq 3$, it then follows that $G^{-}=G_{k}^{-}$. Thus $G^{-} \sqsubset \Lambda_{k-1}^{c}$ and consequently $G \sqsubset \Lambda_{k}^{c}$, so that

$$
\text { (5.2) is implied by } \lim _{L \rightarrow \infty} \mu\left(\left\{\text { there is } k: M_{k}^{-}=\emptyset, \Lambda_{k} \sqsupset \Lambda^{\prime}\right\}\right)=1
$$


The random times $s_{j}$.

We will prove (5.5) by successive approximations, first showing that $\left|M_{k}^{-}\right|$cannot be too large, too often. Call

$$
a(j)=d-1-j+\frac{j}{2 d}, \quad j=1, . ., d
$$

and, setting $s_{0}=0$, define the "random times" $s_{j}, j=1, . ., d$, as

$$
s_{j}= \begin{cases}\min \left\{k:\left|M_{k}^{-}\right| \leq L^{a(j)}\right\}, & \text { if the set is non empty } \\ +\infty & \text { otherwise }\end{cases}
$$

Since $a(j)$ is decreasing, $s_{j} \geq s_{j-1}, j=1, . ., d$. Moreover we can read off from $G_{k}^{-}(q)$ which are the values of $s_{j}$ which are not larger than $k$, if any. Thus there exists a family of sets $K_{j, k}^{0}$ so that

$$
\left\{s_{j}=k\right\} \quad \Leftrightarrow \quad\left\{G_{k}^{-} \in K_{j, k}^{0}\right\}
$$

For $L$ sufficiently large, if $s_{d}<\infty$ then $s_{d}=\min \left\{k \geq s_{d-1}: M_{k}^{-}=\emptyset\right\}$. Thus, by choosing a suitably small constant $b$, we conclude that in the set $\left\{s_{j+1}-s_{j} \leq b L, j<d\right\}$, $\Lambda_{s_{d}} \sqsupset \Lambda^{\prime}$ and, for $L$ large enough, $M_{s_{d}}^{-}=\emptyset$. Recalling (5.5), the statement we want to prove, namely that $\mu \in \mathcal{G}_{\lambda}^{+}$, then follows from

$$
\lim _{L \rightarrow \infty} \mu\left(\left\{s_{j+1}-s_{j}>b L\right\} \sqcap\left\{s_{j} \leq j b L\right\}\right)=0, \quad j=0, . ., d-1
$$

We shorthand

$$
\left\{|q|_{\Delta} \leq L^{\delta}\right\}=\left\{q:\left|q_{x}\right| \leq L^{\delta}, \text { for all } x \in \Delta\right\}, \quad \delta>0, \Delta \sqsubset \mathbb{Z}^{d}
$$

Since $\mu$ is tempered, for any $\delta>0$,

$$
\lim _{L \rightarrow \infty} \mu\left(\left\{|q|_{\Lambda_{0}} \leq L^{\delta}\right\}\right)=1
$$

In fact

$$
\left.\mu\left(\left\{|q|_{\Lambda_{0}}>L^{\delta}\right\}\right) \leq L^{d} \sup _{x \in \Lambda_{0}} \mu\left(\left|q_{x}\right|>L^{\delta}\right)\right)
$$

and then (5.11) follows from (2.12), with $n$ large enough.

By (5.11), we can replace the condition (5.9) by

$$
\lim _{L \rightarrow \infty} \mu\left(\left\{s_{j+1}-s_{j}>b L\right\} \sqcap\left\{s_{j} \leq j b L\right\} \sqcap\left\{|q|_{\Lambda_{0}} \leq L^{\delta}\right\}\right)=0, \quad j=0, . ., d-1
$$

or, for the generic $j \in\{0, . ., d-1\}$ and with $K_{j, k}^{0}$ defined in (5.8),

$$
\lim _{L \rightarrow \infty} \sum_{k \leq j b L} \sum_{G_{k}^{-} \in K_{j, k}^{0}} \mu\left(\left\{s_{j+1}-s_{j}>b L\right\} \sqcap\left\{G_{k}^{-}(q)=G_{k}^{-}\right\} \sqcap\left\{|q|_{\Lambda_{0}} \leq L^{\delta}\right\}\right)=0
$$


The set $\mathcal{B}_{k}$.

We will next suitably modify the set $\left\{s_{j+1}-s_{j}>b L\right\}$. Let $\mathcal{B}_{k}$ be the union of all the maximal connected components of

$$
\left.\left\{x: \theta\left(q_{x}\right)<1\right\} \sqcap\left\{x: \operatorname{dist}\left(x, G_{k}^{-}\right)>3\right)\right\}
$$

which have non empty intersection with $\left\{y: \operatorname{dist}\left(y, M_{k}^{-}\right) \leq 6\right\}$.

The set $G_{h}^{-}, h>k$, contains $G_{k}^{-}$, and the sites $x \in G_{h}^{-} \backslash G_{k}^{-}$, $\operatorname{dist}\left(x, G_{k}^{-}\right) \leq 3$, have distance $\leq 3$ from $M_{k}^{-}$; hence

$$
\left\{x \in G_{h}^{-} \backslash G_{k}^{-}: \operatorname{dist}\left(x, M_{k}^{-}\right)>3\right\} \sqsubset \mathcal{B}_{k}
$$

Since

$$
G_{h}^{-} \backslash G_{k}^{-} \sqsupset \bigsqcup_{k \leq h^{\prime} \leq h} M_{h^{\prime}}
$$

then, in the set $\left\{q: s_{j+1}(q)-s_{j}(q)>b L ; G_{k}^{-}(q)=G_{k}^{-}\right\}, G_{k}^{-} \in K_{j, k}^{0}$,

$$
\left|G_{h}^{-} \backslash G_{k}^{-}\right| \geq b L^{1+a(j+1)}, \quad h \geq k+b L
$$

and by (5.14)

$$
\left|\mathcal{B}_{k}\right| \geq b L^{1+a(j+1)}-50^{d}\left|M_{k}^{-}\right|
$$

On the other hand $\left|M_{k}^{-}\right| \leq L^{a(j)}$ and $a(j+1)+1-a(j)=1 /(2 d)>0$, so that, for $L$ large enough,

$$
\left|\mathcal{B}_{k}\right| \geq \frac{b}{2} L^{1+a(j+1)}
$$

Thus, instead of (5.13) we may prove as well that, for any $j \in\{0, . ., d-1\}$,

$$
\lim _{L \rightarrow \infty} \sum_{k \leq j b L} \sum_{G_{k}^{-} \in K_{j, k}^{0}} \mu\left(\left\{G_{k}^{-}(q)=G_{k}^{-}\right\} \sqcap\left\{|q|_{\Lambda_{0}} \leq L^{\delta}\right\} \sqcap\left\{\left|\mathcal{B}_{k}\right| \geq \frac{b}{2} L^{1+a(j+1)}\right\}\right)=0
$$

Let $G_{k}^{-} \in K_{j, k}^{0}$ in the sequel and call

$$
\Delta_{k}=\left\{x \in \Lambda_{0}: \operatorname{dist}\left(x, G_{k}^{-}\right) \geq 3\right\}, \quad \partial \Delta_{k}=\left\{x \in \Delta_{k}: \operatorname{dist}\left(x, G_{k}^{-}\right)=3\right\}
$$

When $G_{k}^{-}(q)=G_{k}^{-}$, then $\theta\left(q_{x}\right)=1$ for all $x \in \partial \Delta_{k} \sqcap \Lambda_{k}^{c}$; thus

$$
\left\{G_{k}^{-}(q)=G_{k}^{-}\right\}=K_{j, k} \sqcap\left\{\theta\left(q_{x}\right)=1, x \in \partial \Delta_{k} \sqcap \Lambda_{k}^{c}\right\}
$$

where

$$
K_{j, k}=\left\{\theta\left(q_{x}\right)<1, x \in G_{k}^{-}\right\} \sqcap\left\{\theta\left(q_{x}\right)=1, x \in \Lambda_{k}^{c}, \operatorname{dist}\left(x, G_{k}^{-}\right) \in\{1,2\}\right\}
$$


so that $K_{j, k} \in \mathcal{F}_{\Delta_{k}^{c} \sqcap \Lambda_{k}^{c}}$. We then have

$$
\begin{gathered}
\mu\left(\left\{G_{k}^{-}(q)=G_{k}^{-}\right\} \sqcap\left\{|q|_{\Lambda_{0}} \leq L^{\delta}\right\} \sqcap\left\{\left|\mathcal{B}_{k}\right| \geq \frac{b}{2} L^{1+a(j+1)}\right\}\right) \\
=\int_{K_{j, k} \sqcap\left\{\left|q^{\prime}\right|_{\Lambda_{0} \backslash \Delta_{k}} \leq L^{\delta}\right\}} \mu\left(d q^{\prime}\right) \mu_{\lambda, \Delta_{k}}^{\left(q^{\prime}\right)}\left(\left\{\theta\left(q_{x}\right)=1, x \in \partial \Delta_{k} \sqcap \Lambda_{k}^{c}\right\}\right. \\
\left.\sqcap\left\{|q|_{\Delta_{k}} \leq L^{\delta}\right\} \sqcap\left\{\left|\mathcal{B}_{k}\right| \geq \frac{b}{2} L^{1+a(j+1)}\right\}\right)
\end{gathered}
$$

To bound the last term on the r.h.s. of (5.20), we write it as a fraction of two partition functions:

$$
\mu_{\lambda, \Delta_{k}}^{\left(q^{\prime}\right)}\left(\theta=1 \text { on } \partial \Delta_{k} \sqcap \Lambda_{k}^{c} ;|q|_{\Delta_{k}} \leq L^{\delta} ;\left|\mathcal{B}_{k}\right| \geq \frac{b}{2} L^{1+a(j+1)}\right)=\frac{N_{\lambda, \Delta_{k}}^{\left(q^{\prime}\right)}}{Z_{\lambda, \Delta_{k}}^{\left(q^{\prime}\right)}}
$$

where

\section{Reduction to + boundary conditions.}

By using (2.15) we will replace $N_{\lambda, \Delta_{k}}^{\left(q^{\prime}\right)}$ by a new partition function with + boundary conditions. Calling boundary sites, the sites $x \in \Delta_{k}^{c}: \operatorname{dist}\left(x, \Delta_{k}\right) \leq 2$, we want to change $q^{\prime}$ at all the boundary sites where $\theta<1$ and also to impose that $\theta=1$ on the whole $\partial \Delta_{k}$ and not only on $\partial \Delta_{k} \sqcap \Lambda_{k}^{c}$, as in the constraint of the partition function defining $N_{\lambda, \Delta_{k}}^{\left(q^{\prime}\right)}$. The whole thing works because the overall number of sites in this operation is "small", as we are going to see.

The boundary sites have distance equal to 1 or to 2 from $G_{k}^{-}$, hence, by the definition of $G_{k}^{-}, \theta=1$ on all such sites which are not in $\Lambda_{k}$. Thus the new b.c. $q^{\prime+}$ is defined in such a way that $q_{x}^{\prime+}=q_{x}^{\prime}$ for all $x \notin A_{k}, A_{k}=\left\{y \in \Delta_{k}^{c} \sqcap \Lambda_{k}\right.$ : $\left.\operatorname{dist}\left(y, \Delta_{k}\right) \leq 2\right\}$; while $\theta\left(q_{x}^{\prime+}\right)=1$ for all $x \in A_{k}$. Since $\left|\partial \Delta_{k} \sqcap \Lambda_{k}\right| \leq 50^{d}\left|M_{k}^{-}\right|$, by (2.15),

$$
N_{\lambda, \Delta_{k}}^{\left(q^{\prime}\right)} \leq e^{c\left(\beta \ell^{d}\right) 50^{d}\left|M_{k}^{-}\right| L^{\delta n}} Z_{\lambda, \Delta_{k}}^{\left(q^{+}\right)}\left(\theta=1 \text { on } \partial \Delta_{k} ;\left|\mathcal{B}_{k}\right| \geq \frac{b}{2} L^{1+a(j+1)}\right)
$$

where on the r.h.s. we have dropped the condition $|q|_{\Delta_{k}} \leq L^{\delta}$. The r.h.s. is a + partition function in the sense of (2.8) and we have

$$
\frac{Z_{\lambda, \Delta_{k}}^{\left(q^{\prime+}\right)}\left(\theta=1 \text { on } \partial \Delta_{k} ;\left|\mathcal{B}_{k}\right| \geq \frac{b}{2} L^{1+a(j+1)}\right)}{Z_{\lambda, \Delta_{k}}^{\left(q^{\prime+}\right)}\left(\theta=1 \text { on } \partial \Delta_{k}\right)}=\mu_{\lambda, \Delta_{k}}^{\left(+, q^{\prime+}\right)}\left(\left|\mathcal{B}_{k}\right| \geq \frac{b}{2} L^{1+a(j+1)}\right)
$$

The r.h.s. will give the small factor which makes the sum in (5.16) convergent and infinitesimal with $L$, but let us first readjust the partition function in the denominator of the 
fraction on the 1.h.s. of (5.23):

$$
\frac{Z_{\lambda, \Delta_{k}}^{\left(q^{++}\right)}\left(\theta=1 \text { on } \partial \Delta_{k}\right)}{Z_{\lambda, \Delta_{k}}^{\left(q^{\prime+}\right)}\left(\theta=1 \text { on } \partial \Delta_{k},|q|_{\Delta_{k}} \leq L^{\delta}\right)} \leq\left(1-L^{d} \sup _{x \in \Delta_{k}} \mu_{\lambda, \Delta_{k}}^{\left(+, q^{\prime+}\right)}\left(\left|q_{x}\right|>L^{\delta}\right)\right)^{-1}
$$

By (2.12), for $L$ large enough, the last factor is smaller than 2 and, using again (2.15), we get

$$
Z_{\lambda, \Delta_{k}}^{\left(q^{+}\right)}\left(\theta=1 \text { on } \partial \Delta_{k}\right) \leq 2 e^{c\left(\beta \ell^{d}\right) 50^{d}\left|M_{k}^{-}\right| L^{\delta n}} Z_{\lambda, \Delta_{k}}^{\left(q^{\prime}\right)}\left(\theta=1 \text { on } \partial \Delta_{k},|q|_{\Delta_{k}} \leq L^{\delta}\right)
$$

hence, going back to (5.20) and with $c^{\prime}$ a suitable constant,

$$
\begin{gathered}
\mu\left(\left\{G_{k}^{-}(q)=G_{k}^{-}\right\} \sqcap\left\{|q|_{\Lambda_{0}} \leq L^{\delta}\right\} \sqcap\left\{\left|\mathcal{B}_{k}\right| \geq \frac{b}{2} L^{1+a(j+1)}\right\}\right) \\
\leq e^{c^{\prime}\left(\beta \ell^{d}\right)\left|M_{k}^{-}\right| L^{\delta n}} \int_{K_{j, k} \sqcap\left\{|q|_{\Lambda_{0} \backslash \Delta_{k}} \leq L^{\delta}\right\}} \mu\left(d q^{\prime}\right) \mu_{\lambda, \Delta_{k}}^{\left(q^{\prime}\right)}\left(\theta=1 \text { on } \partial \Delta_{k}\right) \\
\times \mu_{\lambda, \Delta_{k}}^{\left(+, q^{+}\right)}\left(\left|\mathcal{B}_{k}\right| \geq \frac{b}{2} L^{1+a(j+1)}\right)
\end{gathered}
$$

$$
\text { The probability that }\left|\mathcal{B}_{k}\right| \geq \frac{b}{2} L^{1+a(j+1)}
$$

By the definition of $\mathcal{B}_{k}$, a maximal connected component of $\mathcal{B}_{k}$ is the layer attached to a plus contour $\Gamma$ whose spatial support has non empty intersection with $F:=\{y$ : $\left.\operatorname{dist}\left(y, M_{k}^{-}\right) \leq 6\right\}$. Let $\underline{\Gamma}=\left(\Gamma_{1}, . ., \Gamma_{n}\right)$ be the collection of all such plus contours, then, by Theorem 4.5,

$$
\begin{aligned}
& \mu_{\lambda, \Delta_{k}}^{\left(+, q^{\prime+}\right)}\left(\left|\mathcal{B}_{k}\right| \geq \frac{b}{2} L^{1+a(j+1)}\right) \sum_{\substack{\Gamma=\left(\Gamma_{1}, \ldots, \Gamma_{n}\right) \\
\operatorname{sp}\left(\Gamma_{i}\right) \sqcap F \neq \emptyset, 1 \leq i \leq n}} e^{-\beta\left(C_{p} / 8\right)|\underline{\Gamma}|} \sum_{\substack{V_{i}, 1 \leq i \leq n \\
\sum_{i} V_{i} \geq \frac{b}{2} L^{1+a(j+1)}}} \prod_{i} e^{-\omega V_{i}} \\
& \leq e^{-\omega(b / 4) L^{1+a(j+1)}} \sum_{\substack{\Gamma=\left(\Gamma_{1}, \ldots, \Gamma_{n}\right) \\
\operatorname{sp}\left(\overline{\Gamma_{i}}\right) \sqcap F \neq \emptyset, 1 \leq i \leq n}} e^{-\beta\left(C_{p} / 8\right)|\underline{\Gamma}|}\left[1-e^{-\omega / 2}\right]^{-n}
\end{aligned}
$$

where we have used (4.23) for the fat contours in $\underline{\Gamma}$ and (4.4) for the slim ones. The number $n$ of contours cannot exceed $|F|$ so that the above is bounded by

$$
e^{-\omega(b / 4) L^{1+a(j+1)}}\left[1-e^{-\omega / 2}\right]^{-|F|} \prod_{x \in F}\left(1+\sum_{\Gamma: \operatorname{sp}(\Gamma) \ni x} e^{-\left(C_{p} / 8\right)|\Gamma|}\right)
$$

For $C_{P}$ as large as required by Lemma 3.1,

$$
\sum_{\Gamma: \operatorname{sp}(\Gamma) \ni x} e^{-\beta\left(C_{p} / 8\right)|\Gamma|} \leq \kappa e^{-C_{P} / 8}
$$


and since $|F| \leq c\left|M_{k}\right| \leq c^{\prime} L^{a(j)}$, we get

$$
\begin{aligned}
\mu_{\lambda, \Delta_{k}}^{\left(+, q^{+}\right)}\left(\left|\mathcal{B}_{k}\right| \geq\right. & \left.\frac{b}{2} L^{1+a(j+1)}\right) \\
& \leq \exp \left\{-\omega(b / 4) L^{1+a(j+1)}+c^{\prime \prime} L^{a(j)}\right\} \leq \exp \left\{-\omega(b / 8) L^{1+a(j+1)}\right\}
\end{aligned}
$$

for $L$ large enough, because

$$
a(j)=d-1-j+\frac{j}{2 d}, \quad 1+a(j+1)=d-1-j+\frac{j+1}{2 d}
$$

Conclusions.

To prove (5.13), we write

$$
\begin{aligned}
& \text { l.h.s. of }(5.13) \leq \lim _{L \rightarrow \infty} \sum_{k \leq j b L} \sum_{G_{k}^{-} \in K_{j, k}^{0}} \exp \left\{c^{\prime}\left(\beta \ell^{d}\right)\left|M_{k}^{-}\right| L^{\delta n}-\omega(b / 8) L^{1+a(j+1)}\right\} \\
& \times \int_{K_{j, k} \sqcap|q|_{\Lambda_{0} \backslash \Delta_{k} \leq L^{\delta}}} \mu\left(d q^{\prime}\right) \mu_{\lambda, \Delta_{k}}^{\left(q^{\prime}\right)}\left(\theta=1 \text { on } \partial \Delta_{k}\right)
\end{aligned}
$$

By (5.7), $\left|M_{k}^{-}\right| \leq L^{a(j)}$, and with such a bound, the first factor on the r.h.s. becomes independent of $k$. Since

$$
\int_{K_{j, k} \sqcap|q|_{\Lambda_{0} \backslash \Delta_{k}} \leq L^{\delta}} \mu\left(d q^{\prime}\right) \mu_{\lambda, \Delta_{k}}^{\left(q^{\prime}\right)}\left(\theta=1 \text { on } \partial \Delta_{k}\right) \leq \mu\left(\left\{G_{k}^{-}(q)=G_{k}^{-}\right\}\right)
$$

we have a sum of probabilities of disjoint events, hence

$$
\text { l.h.s. of }(5.13) \leq \lim _{L \rightarrow \infty} e^{c^{\prime} L^{a(j)}\left(\beta \ell^{d}\right) L^{n \delta}-\omega(b / 8) L^{1+a(j+1)}}=0
$$

if $\delta$ is chosen small enough.

\section{The LMP model}

As an example of the possible applications of Theorem 2.1, we consider the LMP model for phase transitions in the continuum, $[\mathbf{9}]$. Analogous considerations apply to Ising systems with Kac potentials, as in [2] and [4].

\section{The LMP model}

As already mentioned in Section 2, the variable $q_{x}$ in the LMP model is a particle configuration in the cell $C_{x}, C_{x}$ being a cube of a partition $\mathcal{D}^{\left(\ell_{+, \gamma}\right)}$ of $\mathbb{R}^{d}$ which will be described later. $\left|q_{x}\right|$ denotes the number of particles in $q_{x}$ and $|q|$ the total number of particles in the configuration $q$. 
The LMP energy of a finite particle configuration $q=\left(\ldots, r_{i}, ..\right)$ is

$$
H_{\gamma, \lambda, \beta}(q)=\int_{\mathbb{R}^{d}} \beta e_{\lambda}\left(j_{\gamma} * q\right) d r
$$

where, $\gamma>0$ is the Kac scaling parameter,

$$
\begin{gathered}
e_{\lambda}(x)=-\lambda x-\frac{x^{2}}{2}+\frac{x^{4}}{4 !} \\
j_{\gamma} * q(r)=\sum_{r_{i} \in q} j_{\gamma}\left(r, r_{i}\right)
\end{gathered}
$$

with

$$
j_{\gamma}\left(r, r^{\prime}\right):=\gamma^{d} j\left(\gamma r, \gamma r^{\prime}\right)
$$

and $j\left(r, r^{\prime}\right)$ a translational invariant, smooth, symmetric, probability kernel supported by $\left|r-r^{\prime}\right| \leq 1$.

In the language of Section 2,

$$
\alpha_{x}(q)=\frac{\left|q \sqcap C_{x}\right|}{\beta \ell^{d}}, \quad \ell^{d}=\left|C_{x}\right|
$$

while the chemical potential $\lambda$ above differs from $\lambda$ in Section 2 by an additive constant, $\lambda \rightarrow \lambda-\lambda_{\beta, \gamma}$, see the paragraph "Peierls estimates" below.

\section{Mean field}

The mean field limit, formally corresponding to $\gamma=0$, is described by the free energy density

$$
f_{\lambda, \beta}(x)=e_{\lambda}(x)-\frac{s(x)}{\beta}
$$

where $s(\cdot)$ is the entropy density

$$
s(x)=-x(\log x-1)
$$

For $\beta<\beta_{c}=(3 / 2)^{3 / 2}, f_{\lambda, \beta}^{\prime \prime}(x)>0$ for all $\lambda$ and $x$. For any $\beta>\beta_{c}$ there is a unique value of $\lambda, \lambda=\lambda_{\beta}$, where $f_{\lambda_{\beta}, \beta}$ has two distinct minimizers, $\rho_{\beta}^{ \pm}$, elsewhere the minimizer is unique. Minimizers are solutions of the mean field equation $f_{\lambda, \beta}^{\prime}(x)=0$ which can be written as

$$
x=K_{\beta}(x):=e^{-\beta e_{\lambda_{\beta}}^{\prime}(x)}
$$

It has three solutions: $0<\rho_{\beta}^{-}<\rho_{\beta}^{0}<\rho_{\beta}^{+}$, and

$$
f_{\lambda_{\beta}, \beta}^{\prime \prime}\left(\rho_{\beta}^{ \pm}\right)>0
$$

There is $\beta_{0}>\beta_{c}$ so that

$$
K_{\beta}^{\prime}\left(\rho_{\beta}^{+}\right) \in(-1,1) \quad \text { for all } \beta \in\left(\beta_{c}, \beta_{0}\right)
$$

while $K_{\beta}^{\prime}\left(\rho_{\beta}^{-}\right) \in(0,1)$ for all $\beta>\beta_{c}$. Following LMP we restrict to $\beta \in\left(\beta_{c}, \beta_{0}\right)$. 


\section{Contours}

Contours are defined using two scales,

$$
\ell_{ \pm, \gamma}=\gamma^{-(1 \pm \epsilon)}
$$

where $\epsilon>0$ is a "very small" parameter. We then denote by $\mathcal{D}^{\left(\ell_{ \pm, \gamma}\right)}$ two partitions of $\mathbb{R}^{d}$ into cubes of side $\ell_{ \pm, \gamma}$, supposing $\mathcal{D}^{(1)}$ finer than $\mathcal{D}^{\left(\ell_{-, \gamma}\right)}$ finer than $\mathcal{D}^{\left(\ell_{+, \gamma}\right)}$. Thus the cell length $\ell$ of the previous sections is $\ell=\ell_{+, \gamma}=\gamma^{-(1+\epsilon)}$ in the present model.

We then define

$$
\eta^{\left(\zeta, \ell_{-, \gamma}\right)}(q ; r)= \begin{cases} \pm 1 & \text { if }\left|\ell_{-, \gamma}^{-d}\right| q \sqcap C_{r}^{\left(\ell_{-, \gamma}\right)}\left|-\rho_{\beta, \pm}\right| \leq \zeta \\ 0 & \text { otherwise }\end{cases}
$$

and, analogously to $(2.2)$, denoting by $Y$ the centers of all the cubes of $\mathcal{D}^{\left(\ell_{-, \gamma}\right)}$ contained in the cube of $\mathcal{D}^{\left(\ell_{+, \gamma}\right)}$ labelled by $C_{x}$,

$$
\theta\left(q_{x}\right)=\prod_{y \in Y} \mathbf{1}_{\eta^{\left(\zeta, \ell_{-, \gamma}\right)}(q ; y)=1}-\prod_{y \in Y} \mathbf{1}_{\eta^{\left(\zeta, \ell_{-, \gamma}\right)}(q ; y)=-1}
$$

Peierls estimates

Given $\beta \in\left(\beta_{c}, \beta_{0}\right)$ there are $c>0, \zeta>0, \epsilon>0, \gamma^{*}>0$ and $\lambda_{\beta, \gamma}, \gamma \leq \gamma^{*}$, so that the bound (2.9) holds for the Hamiltonian $H_{\gamma, \lambda_{\beta, \gamma}, \beta}$ (which plays the role of the energy $H_{0}$ of Section 2) with

$$
C_{p}=c \zeta^{2} \ell_{-, \gamma}^{d}
$$

with the contours defined using $\zeta>0$ and $\epsilon>0$.

\section{Stability conditions}

Denoting by $H_{\gamma, \lambda, \beta}\left(q \mid q^{\prime}\right)$ the energy of the configuration $q$ in interaction with the configuration $q^{\prime}$, there is a constant $b=b(\lambda, \beta)$ so that

$$
H_{\gamma, \lambda, \beta}\left(q \mid q^{\prime}\right) \geq b|q|
$$

uniformly in $\gamma$ and $q^{\prime}$. From such a strong property, the stability estimates of Section 2 easily follow. Also the cut and paste condition (2.16) is easily seen to hold with $n=4$.

\section{Uniqueness regime}

By applying Theorem 2.1 we deduce that for $\gamma$ small enough, any DLR measure (in the LMP model all DLR measures are stable) is in $\mathcal{G}^{\text {sign of }\left(\lambda-\lambda_{\beta, \gamma}\right)}$ for

$$
0<\left|\lambda-\lambda_{\beta, \gamma}\right| \leq \hat{\lambda}
$$

with $\hat{\lambda}=\hat{c} \gamma^{2 \epsilon}$. This complements the result in [9] about coexistence of phases at $\lambda=\lambda_{\beta, \gamma}$. 
A more accurate analysis would show that $\mathcal{G}^{\text {sign of }\left(\lambda-\lambda_{\beta, \gamma}\right)}$ consists of a single element if $\lambda \neq \lambda_{\beta, \gamma}$, while at the coexistence point, each $\mathcal{G}^{ \pm}$has only one element.

An extension of the methods used in $[\mathbf{3}]$ would allow to prove that uniqueness extends to the region $\left|\lambda-\lambda_{\beta, \gamma}\right|>\hat{\lambda}$.

\section{Acknowledgments}

We are indebted to A. Mazel and A. van Enter for helpful discussions. The Weierstrass Institute in Berlin and the Departments of Mathematics of the Universities of Prague and Roma Tor Vergata have hosted several meetings among the Authors, making possible the collaboration which has led to the present paper.

\section{References}

[1] F. Baffioni, I. Merola, E. Presutti: The liquid and vapor phases in particle models with Kac potentials. Markov Proc. and Rel. Fields, 8 271-318, (2002).

[2] A. Bovier, M. Zahradník: The low temperature phase of Kac-Ising models J. Stat. Phys. 87 311-332, (1997).

[3] P. Buttà, I. Merola, E. Presutti: On the validity of the van der Waals theory in Ising systems with long range interactions Markov Proc. and Rel. Fields 3 63-88, (1997)

[4] M. Cassandro, E. Presutti: Phase transitions in Ising systems with long but finite range interactions. Markov Proc. Related Fields 2, no. 2, 241-262 (1966).

[5] H.A.M. Daniëls, A.C.D. van Enter: Differentiability properties of the pressure in lattice systems. Comm. Math. Phys. 71 no. 1, 65-76, (1980)

[6] S. Friedli, Ch. Pfister: Non-analyticity in the van der Waals limit. Preprint. To appear in J. Stat. Phys. (2003).

[7] R.B. Israel: Generic triviality of phase diagrams in spaces of long-range interactions. Comm. Math. Phys. 106, no. 3, 459-466 (1986) 
[8] J. L. Lebowitz, A. Mazel: On the Uniqueness of Gibbs States in the Pigorov-Sinai Theory Comm. Math. Phys. 189 311-321, (1997)

[9] J. L. Lebowitz, A. Mazel, E. Presutti: Liquid-vapor phase transitions for systems with finite-range interactions. J. Statist. Phys. 94 no. 5-6, 955-1025, (1999).

[10] D. Ruelle: Rigorous results in Statistical Mechanics. Benjamin, (1969)

[11] D. Ruelle: Some ill formulated problems on regular and messy behavior in statistical mechanics and smooth dynamics for which I would like the advice of Yacha Sinai. J. Stat. Phys. 108, (2002)

[12] Ya.G.Sinai: Theory of phase transitions: rigourous results "Nauka" Moskow, 208 pp. (1980)- English translation Pergamon, Oxford-Elmsford, viii + 150 pp.(1982)

[13] A. Sokal: More surprises in the general theory of lattice systems. Comm. Math. Phys. 86, no. 3, $327-336,(1982)$

[14] A.C.D. van Enter: A note on the stability of phase diagrams in lattice systems Comm. Math. Phys. 79, no. 1, 25-32 (1981)

Anton Bovier, Weierstrass-Institute for Applied Analysis and Stochastics, MohrenStrasse 39, 10117 Berlin, Germany

E-mail address: bovier@wias-berlin.de

immacolata Merola, Dipartimento di Matematica, Università di Roma Tor Vergata, 00133 Roma, Italy

E-mail address: merola@mat.uniroma2.it

Errico Presutti, Dipartimento di Matematica, Università di Roma Tor Vergata, 00133 ROMA, ITALY

E-mail address: presutti@mat.uniroma2.it

Miloš Zahradník, Department of Mathematical Analysis, Faculty of Mathematics and Physics, Charles University, 18600 Prague, Sokolovská 83

E-mail address: mzahrad@karlin.mff.cuni.cz 